\title{
Specific Trans-Synaptic Interaction with Inhibitory Interneuronal Neurexin Underlies Differential Ability of Neuroligins to Induce Functional Inhibitory Synapses
}

\author{
Kensuke Futai, ${ }^{1,2}$ Christopher D. Doty, ${ }^{1 *}$ Brian Baek, ${ }^{2 \star}$ Jubin Ryu, $^{2}$ and Morgan Sheng ${ }^{2}$ \\ ${ }^{1}$ Brudnick Neuropsychiatric Research Institute, and Department of Psychiatry, University of Massachusetts Medical School, Worcester, Massachusetts \\ 01604 and ${ }^{2}$ The Picower Institute for Learning and Memory, Massachusetts Institute of Technology, Cambridge, Massachusetts 02139
}

Synaptic transmission depends on the matching and alignment of presynaptically released transmitters and postsynaptic neurotransmitter receptors. Neuroligin (NL) and Neurexin (Nrxn) proteins are trans-synaptic adhesion molecules that are important in validation and maturation of specific synapses. NL isoforms NL1 and NL2 have specific functional roles in excitatory and inhibitory synapses, respectively, but the molecular basis behind this distinction is still unclear. We show here that the extracellular domain of NL2 confers its unique ability to enhance inhibitory synaptic function when overexpressed in rat hippocampal pyramidal neurons, whereas NL1 normally only promotes excitatory synapses. This specificity is conferred by presynaptic Nrxn isoforms, as NL1 can also induce functional inhibitory synapse connections when the presynaptic interneurons ectopically express an Nrxn isoform that binds to NL1. Our results indicate that trans-synaptic interaction with differentially expressed presynaptic Nrxns underlies the distinct functions of NL1 and NL2, and is sufficient to induce functional inhibitory synapse formation.

\section{Introduction}

Excitatory (glutamatergic) and inhibitory (GABAergic) neurons send axons to their target neurons and form functional synaptic connections where postsynaptic receptors for glutamate and GABA are appropriately concentrated (Yamagata et al., 2003). However, the exact mechanisms that coordinate the alignment of presynaptic transmitter type with its cognate postsynaptic neurotransmitter receptor are not elucidated. Postsynaptically expressed Neuroligins (NLs; NL1-4) trans-synaptically bind Neurexins (Nrxns) [ $\alpha$ Nrxn1-3 (long form) or $\beta$ Nrxn $1-3$ (short)] and differentially regulate inhibitory and excitatory synapse function (Südhof, 2008). NL1 and NL2 are primarily situated in excitatory and inhibitory synapses, respectively (Song et al., 1999; Graf et al., 2004; Varoqueaux et al., 2004), and regulate corresponding synaptic function (Chubykin et al., 2007; Futai et al., 2007; Gibson et al., 2009; Blundell et al., 2010). While this indicates that NL1 and NL2 can distinguish between GABAergic and glutamatergic presynaptic inputs, the molecular mechanisms underlying the NL-

\footnotetext{
Received April 13, 2012; revised Dec. 13, 2012; accepted Jan. 7, 2013.

Author contributions: K.F. and M.S. designed research; K.F., C.D.D., B.B., and J.R. performed research; K.F. contributed unpublished reagents/analytic tools; K.F. analyzed data; K.F. and M.S. wrote the paper.

K.F. was supported by UMASS start-up funds and Whitehall Foundation. We thank Dieter Edbauer, Nakagawa Terunaga, Maria Morabito, and Yasunori Hayashi for valuable advice. We thank Drs. Elaine Budreck, Hiroki Taniguchi, and Peter Scheiffele for providing neuroligin and neurexin cDNA clones.

*C.D.D. and B.B. contributed equally to this work.

The authors declare no competing financial interests.

Correspondence should be addressed to Dr. Kensuke Futai, Department of Psychiatry, Brudnick Neuropsychiatric Research Institute, University of Massachusetts Medical School, 303 Belmont Street, Worcester, MA 01604-1676. E-mail: Kensuke.Futai@umassmed.edu.

M. Sheng's present address: Department of Neuroscience, Genentech Inc., South San Francisco, CA 94080.

DOI:10.1523/JNEUROSCI.1811-12.2013

Copyright $\odot 2013$ the authors $\quad 0270-6474 / 13 / 333612-12 \$ 15.00 / 0$
}

dependent formation of functional excitatory and inhibitory synapses are poorly understood. It has been postulated that specific interactions with postsynaptic molecules may determine the differential subcellular localization of NL isoforms (Barrow et al., 2009; Poulopoulos et al., 2009). However, the postsynaptic molecules that determine the differential localization and function of NLs are still not identified.

Coculture studies have revealed that overexpression of $\beta \mathrm{Nrxn}$ isoforms in non-neuronal cells induced the aggregation of both inhibitory and excitatory postsynaptic proteins in contacting neurons, while $\alpha$ Nrxns triggered the assembly of inhibitory but not excitatory postsynaptic proteins (Nam and Chen, 2005; Chih et al., 2006; Kang et al., 2008). Moreover, knock-out mice lacking $\alpha$ Nrxns exhibited a reduction in the density of symmetric inhibitory synapses in the brainstem (Missler et al., 2003). Therefore, $\alpha$ Nrxns may have critical roles in inhibitory synapse formation. The study of Nrxn expression in hippocampus indicates region-specific and cell type-specific expression patterns of different Nrxn isoforms (Ullrich et al., 1995), which may limit the combinations of trans-synaptic NL-Nrxn interactions that can occur between particular pairs of contacting neurons. Therefore, the restricted pairing of NL-Nrxn isoforms between particular cell types may impart specificity on the synaptic functions of NL and Nrxn isoforms. For instance, if the Nrxn binding partners of a particular NL isoform are expressed only in inhibitory interneurons, then expression of that NL isoform in the postsynaptic neuron might be expected to promote inhibitory, but not excitatory synaptic function. This concept of synaptic specificity mediated by specific NL-Nrxn interactions has not been directly tested in neurons.

We report here that the extracellular domain of NL1 and NL2 confer the differential activities of these NLs on inhibitory synap- 
tic transmission, and that $\beta$ Nrxns are abundantly expressed in excitatory neurons compared with inhibitory neurons. Furthermore, we demonstrate that even NL1, which is specific for excitatory synapses, can induce functional inhibitory synapses when the NL1 binding partner, $\beta \mathrm{Nrxn} 1$, is introduced in presynaptic interneurons. Our results indicate that the NL-Nrxn interaction is sufficient to promote functional inhibitory synapses and that the differential role of NL1 and NL2 on inhibitory synaptic function is attributable to the low abundance of the primary NL1 ligand, $\beta \mathrm{Nrxn}$, in presynaptic interneurons.

\section{Materials and Methods}

Molecular biology

Expression and shRNA vectors. The expression and shRNA vectors have been reported previously for hemagglutinin (HA)-tagged mouse (m) NL1AB, mNL2A, $\mathrm{m} \beta \operatorname{Nrxn} 1(4 \pm)$, rat (r) $\alpha \operatorname{Nrxn1} 14-)$, and rNL2 shRNA (Scheiffele et al., 2000; Chih et al., 2005; Chih et al., 2006). HA-tagged $\mathrm{m} \beta \mathrm{Nrxn} 2$ (4-) and $\mathrm{m} \beta \mathrm{Nrxn} 3$ (4-) were gifts from Dr. Scheiffele (Biozentrum, University of Basel, Basel, Switzerland). Short hairpin sequences used for the knockdown experiments of NL2 were as follows: rNL2-shRNA: TGGAGCAAGTTC AACAGCAATTCAAGAGATTGCTGTTGAACTTGCTCCTTT TTTC. Enhanced green fluorescent protein (EGFP), DsRed2 (Clontech), and TagBFP (Evrogen) were subcloned in the pCAG vector. The chimeric HA-tagged NL1AB with NL2 transmembrane and C terminus (NL1AB/NL2) was made by fusing the $\mathrm{N}$ terminus (1695) of NL1AB (NP 619607) with amino acid (677-836) of NL2A (NP 942562) with a PCR-based method. The chimeric NL2A (1676) with NL1 transmembrane and C terminus domains (696-843) was made in a similar fashion.

Single-cell reverse transcription. Harvesting the cytosol from CA3 and CA1 neurons was performed using the whole-cell patch-clamp technique described below. Patch pipettes were filled with the DEPC-treated internal solution containing the following (in $\mathrm{mM}$ ): $140 \mathrm{~K}$-methanesulfonate, 0.2 EGTA, $2 \mathrm{MgCl}_{2}$, and 10 HEPES, $\mathrm{pH}$ adjusted to 7.3 with $\mathrm{KOH}$. The resistance of the patch electrodes was 2.0-3.0 $\mathrm{M} \Omega$. The glass pipettes were baked at $200^{\circ} \mathrm{C}$ overnight. All other equipment, including electrode holder and tubing, was sprayed by RNase inhibitor (RNase Away) and kept clean before use. RNase inhibitor (1 U/ $\mu$ l; Ambion) was dissolved into internal solution for single-cell quantitative PCR (qPCR). Before filling the electrode with RNase inhibitor-containing solution $(4.5 \mu \mathrm{l})$, the tip of the electrode was always loaded with a small volume of internal solution $(\sim 0.5 \mu \mathrm{l})$ to make a smooth seal formation. Immediately after the establishment of whole-cell recording, the contents of the recorded cell were aspirated into the patch pipette. The nuclei of target cells were not aspirated into the patch pipettes to avoid contamination of genomic DNA. The cytosol of the cell was expelled into an RNase-free $0.5 \mathrm{ml}$ tube (Ambion) containing $15 \mu \mathrm{l}$ of a solution consisting of dNTPs, random hexamer, and oligo-dT15 (BioRad, iScript cDNA synthesis kit). After expelling the contents of the patch pipette into the reaction tube (total $20 \mu \mathrm{l}$ ), the mixture was incubated at $25^{\circ} \mathrm{C}$ for $5 \mathrm{~min}, 42^{\circ} \mathrm{C}$ for $3 \mathrm{~h} 30 \mathrm{~min}$, and $85^{\circ} \mathrm{C}$ for $5 \mathrm{~min}$.

Diagnostic PCR. The sequences of primers for the diagnostic PCR were designed by Perlprimer or as described previously (Jonas et al., 1999) (see below). The cDNA fragments of diagnostic genes were amplified by two-step PCR. Briefly, a solution containing the TaqDNA polymerase (2.5 U; Bio-Rad), the primer mixtures (a total of 22 primers, 5 pmol each), and template was added. For single-cell qPCR (see Fig. 3), 10\% of total volume of cDNA aliquot $(2 \mu \mathrm{l})$ was used for diagnostic PCR and 90\% (18 $\mu \mathrm{l})$ for the single-cell qPCR (see below, Single-cell qPCR). The thermal cycling was $95^{\circ} \mathrm{C}$ for $15 \mathrm{~min}, 94^{\circ} \mathrm{C}$ for $30 \mathrm{~s}, 60^{\circ} \mathrm{C}$ for 1 $\min$, and $72^{\circ} \mathrm{C}$ for $1 \mathrm{~min}$ for 20 cycles. An aliquot $(3 \mu \mathrm{l})$ of this reaction was added to the second PCR mixture containing one primer pair $(0.1 \mu \mathrm{M}$ each). A total of 35 amplification cycles was performed under the same condition as the first PCR. The second PCR products were separated on $1.5 \%$ agarose gels and the type of neuron was determined. Samples that failed to show expression of two ubiquitous genes, YWHAZ and tubulin, were not used for the single-cell qPCR. The cells that expressed GAD65 and/or GAD67 were defined as inhibitory interneurons and vesicular glutamate transporter 1 (VGluT1)-positive neurons as excitatory neurons. There were no cells expressing both inhibitory and excitatory neuronal markers. The sequence of PCR primers were as follows: GAD65, GenBank accession number, M72422: forward primer, CATCGCATTCACGTCAGAG; reverse primer, GAGACATCAGTAACCCTCCA; GAD67, GenBank accession number, M76177: forward primer, CTCAACTATGTCCGCAAG AC; reverse primer, CCATAAGAACAAACACGGGTG; VGluT1, GenBank accession number, RNU07609: forward primer, ATGT CCACGACCAATGTG; reverse primer, GAGGAACACGTACT GCCA; PV, GenBank accession number, NM 022499: forward primer, GATGACAGACTTGCTCAGC; reverse primer, GAGT GGAGAATTCTTCAACCC; Calb1, GenBank accession number, BC081764: forward primer, AGCTGCAGAACTTGATCCA; reverse primer, CTTGTTTGCTTTCTCTAGCAGG; Calb2, GenBank accession number, BC087603: forward primer, ATCCCA GTTCCTGGAAATCTG; reverse primer, ATACTTCCGCCAAG CCTC; Cck, GenBank accession number, NM $\quad$ 012829: forward primer, GCATCCGAAGATATGAAGTGC; reverse primer, AG TCCCGGTCACTTATCC; Sst, GenBank accession number, NM 012659: forward primer, AGACTCCGTCAGTTTCTGC; reverse primer, GAGAGGGATCAGAGGTCTG; VIP, GenBank accession number, XM 001065820: forward primer, TATGGG CCACCTTCTTCAG; reverse primer, AGACTGCATCAGAGTG TCG; YWHAZ, GenBank accession number, BC094305: forward primer, CGTAGGTCATCTTGGAGGG; reverse primer, CAAT TCCTTTCTTGTCATCACC; Tubulin, GenBank accession number, J00798: forward primer, CTCGCATCCACTTCCC TC; reverse primer, ATGCCCTCACCCACGTAC.

Single-cell $q P C R$. The single-cell cDNA samples identified for neuronal types by diagnostic PCR were further tested by singlecell qPCR. All primer sets and locked nucleic acid (LNA)substituted Taqman probes were designed by the Beacon Designer. We first tested 129 primer sets for Nrxn isoforms ( $\alpha$ Nrxn1: 44 primer sets, $\alpha$ Nrxn2: 26, $\alpha$ Nrxn3: $25, \beta$ Nrxn1: 25 , $\beta$ Nrxn2: $4, \beta$ Nrxn3: 5 ) by SYBR green (Bio-Rad iQ SYBR Green kit) using the dilution series of cDNA prepared from P14 rat hippocampi and the primer sets, which exhibited the highest PCR efficiency with the corresponding Taqman probes, were chosen for the multiplex qPCR. None of the primers of $\beta$ Nrxns designed to span or flank introns exhibited $>80 \%$ PCR efficiency. GAPDH was used as an internal control gene to normalize gene expression levels. The Taqman-based multiplex real-time PCRs were performed in duplicate, and contained cDNA sample $(9 \mu \mathrm{l})$ plus four probes, primer sets ( $\alpha$ - or $\beta$ Nrxn $1-3$ and GAPDH) and multiplex qPCR mix (iQ Multiplex Powermix; Bio-Rad). A total of 40 amplification cycles were performed in a CFX96 Real-Time PCR Detection System (Bio-Rad) consisting of a heating step of $95^{\circ} \mathrm{C}$ for $15 \mathrm{~s}$, followed by annealing and extension at $60^{\circ} \mathrm{C}$ for $50 \mathrm{~s}$. Before amplification, the reaction mixture was held for 3 $\min$ at $95^{\circ} \mathrm{C}$. The relative expression of Nrxns is given by the following: Relative expression $=\left(1+E_{\mathrm{GAPDH}}\right)^{\mathrm{Ct}, \mathrm{GAPDH}} /(1+$ 
$\left.E_{\mathrm{Nrxn}}\right)^{\mathrm{Ct}, \mathrm{Nrxn}} ; E$, efficiency of target amplification (see PCR efficiency) and $\mathrm{Ct}$, threshold cycle for target gene amplification.

The sequence of qPCR primers and probes were as follows: $\alpha$ Nrxn1, GenBank accession number, M96374: forward primer, TG GCTAAAGAAACATACAAATCG; reverseprimer,GCAGAAAAGA GCATCAGATATG; sense probe, 5' Cy5-aabgCctGccAttCaagt cc-IowaBlackRQ-Sp3'; PCR efficiency, 77; $\alpha$ Nrxn2, GenBank accession number, M96376: forward primer, CTGGGCTCTGATGA CTTC; reverse primer, TCAGGACTCTCGAAGGTC; sense probe, 5'HEX-cccAacAcaGccGacc-IowaBlack FQ3'; PCR efficiency, 86; $\alpha$ Nrxn3, GenBank accession number, NM 053817: forward primer, GGAAACCCCAAGAAAGGAAAG; reverse primer, ATCC CGCTGAATGTCCAC; sense probe, 5' Tex615- cggAgcCagAagAaca c-IowaBlackRQ-Sp3'; PCR efficiency, 88 ; $\beta$ Nrxn1, GenBank accession number, M96375: forward primer, CTGGATAGTCCCGC TCAC; reverse primer, GACCTGTAGATTGCAATAGGC; antisense probe, 5'Cy5-atgCttGctGctGcca-IowaBlackRQ-Sp3'; PCR efficiency, 75; $\beta \mathrm{Nrxn} 2$, GenBank accession number, M96377: forward primer, CACGTCCACCACTTCCAC; reverse primer, CCCT TCCCAAAGATGTATGTG; sense probe, 5'HEX-ccaTcgCcaTcaAc cg-IowaBlack FQ3'; PCR efficiency, 83; $\beta$ Nrxn3, cloned by Dr. Hiroki Taniguchi: forward primer, CTCTGTGTGGAGTTCTTCTA ATG; reverse primer, GCCGTGGAAATGGTGTTC; sense probe, 5'Tex615-agcCtcCtcCtcCtcctc-IowaBlackRQ-Sp3'; PCR efficiency, 88; GAPDH, GenBank accession number, NM 017008: forward primer, CGTGTTCCTACCCCCAATG; reverse primer, CTTCAC CACCTTCTTGATGTC; sense probe, 5'6-FAM-tctGacAtgCcgCct g-IowaBlackFQ3'; PCR efficiency, 88. The capital and bold letters in probes are LNAs.

Primary hippocampal neuron culture, transfection, and immunocytochemistry. Primary hippocampal cultures were prepared from the brains of individual rat embryos at embryonic day 19 (either sex) as described previously (Brewer et al., 1993) with some modifications. Hippocampi were dissected at $4^{\circ} \mathrm{C}$ and digested with trypsin, which was inactivated by the addition of fetal calf serum. Tissue was dissociated in Neurobasal/B27 (Invitrogen) medium. Cells were plated onto coverslips (Matsunami) coated with poly-D-lysine $(80 \mu \mathrm{g} / \mathrm{ml}, \mathrm{BD})$ and laminin $(2 \mu \mathrm{g} / \mathrm{ml})$ at a density of 200 cells $/ \mathrm{mm}^{2}$ in Neurobasal medium supplemented with 2\% B27 supplement. To visualize axonal $\beta$ Nrxn1, cultured neurons were transfected with blue fluorescent protein (BFP) on $14 \mathrm{~d}$ in vitro (DIV) using Lipofectamine 2000 (Invitrogen). For immunocytochemistry, neurons were fixed at DIV 14-21 with 4\% paraformaldehyde (PFA). Primary and secondary antibodies were applied in GDB buffer (30 mM phosphate buffer, $\mathrm{pH} 7.4$, containing $0.2 \%$ gelatin, $0.5 \%$ Triton $\mathrm{X}-100$, and $0.45 \mathrm{M} \mathrm{NaCl}$ ).

Antibodies. The following antibodies were used: rabbit antivesicular GABA transporter (VGAT; 1:5000 dilution; Synaptic Systems); guinea pig anti-VGluT1 (1:10000; Synaptic Systems); rabbit anti-parvalbumin (1:2000; SWANT); goat anti-parvalbumin (1: 1000 ; SWANT); mouse anti- $\beta$ Nrxn1 (1:1000, Neuromab); rabbit anti-HA (1:3000; Santa Cruz Biotechnology); mouse anti-GAPDH (1:1000; Millipore Bioscience Research Reagents); secondary Alexa dye-conjugated anti-mouse (Alexa 488), anti-rabbit (Alexa 594), anti-goat (Alexa 405 or 594), and anti-guinea pig (Alexa 647) antibodies (Invitrogen); horseradish peroxidase-conjugated anti-mouse and anti-rabbit (GE Healthcare) antibodies.

Western blotting. Nrxn-transfected HEK293T cells were solubilized in lysis buffer (10 mM Tris, pH 8.0, $200 \mathrm{mM} \mathrm{NaCl}, 1 \%$ Triton X-100, 1\% SDS, protease inhibitors) and loaded onto $8 \%$ SDS-PAGE gels. Crude membrane fraction of rat hippocampi (P14) was isolated as described previously (Carlin et al., 1980). Protein bands were transferred to nitrocellulose membranes
(Bio-Rad). Primary antibodies (1:1000 to 1:3000 dilution) were applied in blocking buffer (20 mM Tris, $\mathrm{pH} 7.4,137 \mathrm{~mm} \mathrm{NaCl}$, $0.1 \%$ Tween 20 , and $1 \%$ bovine serum albumin, $5 \%$ nonfat milk) for $2 \mathrm{~h}$ at room temperature. Secondary antibodies were used at 1:2000 dilution. The signal was detected using an ECL detection kit (PerkinElmer Life Sciences).

Neuronal imaging. Immunostaining of axonal and dendritic segments: confocal images of $512 \times 512$ pixels were taken from hippocampal primary culture with a spinning disk confocal microscope (Nikon; University of Massachusetts Medical School Imaging Core Facility), and $\times 100$ or $\times 40$ objective with sequential acquisition settings. Long narrow processes with varicosities were defined as axons and VGluT1-immunopositive sites were defined as presynaptic terminals of excitatory synapses.

Each image was a $Z$-series projection of $x$ - $y$ images, and taken at $0.2 \mu \mathrm{m}$ depth intervals. Morphometric measurements were made using MetaMorph software (Molecular Devices). The colocalization of $\beta$ Nrxn 1 and VGluT1 or VGAT staining signals were evaluated by dividing the area of $\beta \mathrm{Nrxn} 1$, which was overlapped with VGluT1 or VGAT, by the total area of $\beta$ Nrxn1.

Morphological analysis of spines from cultured hippocampal slices were performed as follows. Three days after biolistic transfection of GFP with or without NLs in organotypic slice cultures, the slices were fixed with $4 \%$ PFA and $4 \%$ sucrose in PBS overnight. The slices were then cryoprotected in $30 \%$ sucrose in $0.1 \mathrm{M}$ phosphate buffer ( $\mathrm{pH}$ 7.4) for $2 \mathrm{~h}$ at room temperature, rapidly frozen on dry ice, thawed in PBS, and stained with GFP antibodies in GDB buffer ( $0.1 \%$ gelatin, $0.3 \%$ TX-100, $450 \mathrm{~mm} \mathrm{NaCl}$, and $32 \% 0.1 \mathrm{M}$ phosphate buffer, $\mathrm{pH}$ 7.4) (McAllister, 2000). Confocal images were obtained using a $63 \times$ objective. Morphometric analysis and quantification were performed using MetaMorph software. All measurements were made in a "blind" manner.

\section{Electrophysiology}

Simultaneous recording from two adjacent hippocampal CA1 pyramidal cells. Organotypic hippocampal slice cultures were prepared from postnatal 6- to 7-d-old rats of either sex as described previously (Nakagawa et al., 2004; Futai et al., 2007). All animal protocols were approved by the Institutional Animal Care and Use Committee at Massachusetts Institute of Technology and University of Massachusetts Medical School. Neurons were transfected using biolistic gene gun (Bio-Rad) at DIV 4-6, and were assayed 3 or $5 \mathrm{~d}$ after transfection of NLs or NL2-shRNA, respectively. A total of $100 \mu \mathrm{g}$ DNA and $10 \mathrm{mg}$ gold particles (1.6 $\mu \mathrm{m}$ diameter) were used to prepare $\sim 50$ bullets. The extracellular solution was as follows (in $\mathrm{mm}$ ): $119 \mathrm{NaCl}, 2.5 \mathrm{KCl}, 4 \mathrm{CaCl}_{2}$, $4 \mathrm{MgCl}_{2}, 26 \mathrm{NaHCO}_{3}, 1 \mathrm{NaH}_{2} \mathrm{PO}_{4}, 11$ glucose, and 0.01 2-chloroadenosine (Sigma), gassed with $5 \% \mathrm{CO}_{2} / 95 \% \mathrm{O}_{2}, \mathrm{pH}$ 7.4, unless otherwise noted. 2-Chloroadenosine, an agonist of the adenosine A1 receptor, was included in the extracellular solution to prevent bursting of the slice culture. Whole-cell voltage-clamp recordings were made simultaneously from a pair of CA1 pyramidal neurons, one transfected (visualized by cotransfecting GFP) and one untransfected neighbor. The patch recording pipettes $(2-4 \mathrm{M} \Omega$ ) were filled with internal solution containing the following (in $\mathrm{mM}$ ): 115 cesium methanesulfonate, $20 \mathrm{CsCl}, 10$ HEPES, $2.5 \mathrm{MgCl}_{2}$, 4 adenosine triphosphate disodium salt, 0.4 guanosine triphosphate trisodium salt, 10 sodium phosphocreatine, and 0.6 EGTA, at pH 7.25 with $\mathrm{CsOH}$. To measure $\mathrm{GABA}_{\mathrm{A}^{-}}$ IPSC and AMPAR-EPSC, NMDAR antagonist (D-APV, 0.05 $\mathrm{mM}$, Ascentscientific) was dissolved into artificial CSF (ACSF) throughout the recording. $\mathrm{GABA}_{\mathrm{A}}$ receptor-mediated IPSC was first measured at $V_{\text {hold }}=0 \mathrm{mV}$. Stimulus strength was set to 
produce an IPSC amplitude of 500-1000 pA in untransfected pyramidal neurons. After obtaining 40 to 50 consecutive stable IPSC responses evoked at $0.2 \mathrm{~Hz}$ with a stimulating electrode placed in the stratum radiatum, picrotoxin $(0.1 \mathrm{mM}$, Sigma) was added to ACSF to eliminate the IPSC. Then, AMPAR-EPSCs were evoked at $V_{\text {hold }}=-60 \mathrm{mV}$ without changing stimulus strength. This stimulus condition evoked $\sim 50 \mathrm{pA}$ of AMPAR-EPSC. The measurement of $\mathrm{GABA}_{\mathrm{A}} \mathrm{R}$-mediated and AMPAR-mediated miniature synaptic current was performed similarly (to measure stimulation-evoked GABA $\mathrm{R}$-PSCs and AMPAR-PSCs) in the presence of tetrodotoxin (TTX; $0.001 \mathrm{mM}$; Tocris Bioscience) in extracellular solution. Events smaller than $5 \mathrm{pA}$ were excluded from the analysis. Experiments and analysis were done blind to the DNA constructs used.

Presynaptic and postsynaptic double whole-cell recording from hippocampal CA1 neurons. Dual whole-cell recordings were performed from presynaptic untransfected interneurons and postsynaptic pyramidal cells expressing GFP with or without NL isoform. Double or triple whole-cell recordings were performed from DsRed 2 and Nrxn isoform expressing presynaptic interneuron, untransfected and GFP with NL expressing postsynaptic pyramidal cells. 2-Chloroadenosine was omitted from the extracellular solution. We used DEPC-treated potassium-based internal solution (substituted potassium for cesium) for whole-cell recording of presynaptic interneurons. Synaptic transmission was evoked by applying two $70 \mathrm{mV}$ depolarization pulse from $-70 \mathrm{mV}$ of $2-4 \mathrm{~ms}$ duration to the presynaptic interneuron in voltage-clamp mode delivered at $0.1 \mathrm{~Hz}$ while recording postsynaptic IPSCs from the excitatory neuron at $0 \mathrm{mV}$. Synaptic connectivity was tested by applying 50 consecutive paired (at $50 \mathrm{~ms}$ interval) stimulations; responses larger than $10 \mathrm{pA}$ observed within $5 \mathrm{~ms}$ after the onset of either of the pulses were counted as evoked unitary $\mathrm{GABA}_{\mathrm{A}} \mathrm{R}$-IPSC. If any evoked response was observed during this period, the pair was considered synaptically connected. Immediately after recording, cytosol of presynaptic interneurons was collected in a recording pipette and expelled into reverse transcription reaction solution from which diagnostic PCR was performed to identify the interneuron type. Experiments were done blind to the DNA constructs used.

Single-cell electroporation to presynaptic interneurons. One day after biolistic gene transfection as described above, slices were transferred to a microscope equipped with differential interference contrast optics and perfused with the extracellular solution with TTX (0.001 mM) but without 2-chloroadenosine. Biolistically transfected NL isoform in CA1 pyramidal neurons was identified by coexpressed EGFP epifluorescence. Inhibitory neurons within $100 \mu \mathrm{m}$ radius of the GFP-positive pyramidal neurons were visually identified and were electroporated with DsRed 2 and Nrxn plasmid-containing solution $(0.1-0.2 \mu \mathrm{g} / \mathrm{ml})$ with $100 \mathrm{~Hz}$ frequency of $1 \mathrm{~V}$ square pulse for 300-700 ms through patch pipette (3-5 M $\Omega$ ) using a single-cell electroporator (Axoporator 800A; Molecular Devices). Plasmids $(0.2 \mu \mathrm{g} / \mu \mathrm{l})$ were dissolved in the same internal solution for single-cell reverse transcription. After electroporation, slices were transferred to the $\mathrm{CO}_{2}$ incubator and cultured under semisterilized condition for $2 \mathrm{~d}$. Note that the transfection duration of NL isoforms in Figures 5 and 6 are $3 \mathrm{~d}$, which is the exact same transfection condition we used in Figures 1 and 2 .

\section{Statistical analysis}

Results are reported as mean \pm SEM. The statistical significance was evaluated by Kruskal-Wallis ANOVA or one-way ANOVA with post hoc Dunnett for multiple comparison, and by Student's $t$ test or Mann-Whitney test for two-group comparison. Statistical significance was set at $p<0.05$.

\section{Results}

\section{NL2 isoforms but not NL1 regulate inhibitory synaptic transmission}

To examine the roles of NL1 and NL2 in excitatory and inhibitory synapse function, we biolistically transfected NL isoforms NL1AB or NL2A [the two major splice forms of NL1 and NL2 expressed in hippocampus (Chih et al., 2006)] in CA1 pyramidal cells of organotypic hippocampal slice cultures (Fig. 1). Simultaneous electrophysiological recordings were made from transfected and neighboring untransfected neurons. CA1 pyramidal cells overexpressing NL1AB showed evoked GABA $\mathrm{A}_{\mathrm{A}} \mathrm{R}$-mediated IPSCs indistinguishable from neighboring, untransfected control neurons, but a marked increase in AMPAR-mediated EPSCs, as reported previously (Fig. 1A,F, for summary) (Chubykin et al., 2007; Futai et al., 2007). In contrast, overexpression of NL2A induced enhancement of IPSC as well as EPSC compared with neighboring untransfected cells (Fig. $1 B, F$ ). The NL2-mediated enhancement of IPSC and EPSC did not require the presence of the alternative splice insertion at site $\mathrm{A}$ (Fig. $1 C, F$ ). We also compared the quantal events of inhibitory and excitatory synaptic responses (mIPSC and mEPSC) in NL-transfected neurons (Fig. 2). Postsynaptic transfection of NL1AB greatly increased mEPSC frequency (but not amplitude) compared with untransfected neurons (Fig. $2 B, C$ ); in contrast, there was no significant effect on mIPSC amplitude or frequency (Fig. 2E,F). NL2A overexpression increased the frequency of both mIPSCs and mEPSCs (Fig. 2, C,F), although there was a modest reduction of mIPSC amplitude (Fig. 2E). Thus NL1 specifically enhanced excitatory synaptic input, whereas NL2 enhanced both excitatory and inhibitory synaptic transmission onto the transfected cell. The above results suggest that the enhancement of EPSC by NL1 and NL2 is due to an increase in the number of functional excitatory synapses, consistent with studies in dissociated neurons (Chih et al., 2005, 2006; Levinson et al., 2005). We measured the spine density of CA1 pyramidal neurons overexpressing NL1AB or NL2A in hippocampal slice culture (Fig. 2G-J). Compared with GFP transfection alone, both NL1AB and NL2A transfection caused a significant increase in spine density, with no change in spine dimensions (Fig. 2H-J), which is in line with NL1 and NL2 having a large effect on enhancement of mEPSC frequency. The stimulatory effect of NL2A on excitatory synapses has been reported in dissociated cultured neurons (Chih et al., 2005, 2006; Levinson et al., 2005) and overexpressed NL2 is reported to show colocalization with PSD-95 (Graf et al., 2004; Levinson et al., 2005), though not consistently (Chubykin et al., 2007).

\section{The extracellular domains of NLs determine the specific synaptic function of NL isoforms}

Inhibitory synaptic function was enhanced by postsynaptic overexpression of NL2A (Fig. 1B,F). Moreover, shRNA-mediated knockdown of endogenous NL2 resulted in selective reduction of IPSC amplitude, with no effect on EPSC (data not shown) [IPSC amplitudes (untransfected: $603.6 \pm 75.5 \mathrm{pA}$; transfected $149.2 \pm$ $24.8 \mathrm{pA} ; p<0.001$ ) and EPSC amplitudes (untransfected: $36.6 \pm$ $7.4 \mathrm{pA}$; transfected $31.8 \pm 7.8 \mathrm{pA} ; p=0.2)$ ]. These results are consistent with another study (Chubykin et al., 2007) and indicate a specific role of postsynaptic NL2 in regulation of inhibitory synaptic input.

Which domain of NL2 mediates its action on inhibitory synapse? Because NL1 has no effect on inhibitory synaptic transmis- 
sion (Fig. $1 A, F$ ), we swapped the NL1AB and NL2A extracellular domains to generate chimeric constructs in which the NL1AB extracellular domain was conjugated to the transmembrane (TM) and intracellular domains of NL2 (NL1AB/ NL2), or the NL2A extracellular domain was fused to the TM and intracellular domains of NL1 (NL2A/NL1). We then measured IPSCs and EPSCs in neurons transfected with these chimeras. NL1AB/ NL2 transfection had no significant effect on IPSC amplitude compared with untransfected neurons (Fig. 1D). In contrast, NL2A/NL1 transfection increased IPSC, similarly to NL2A (Fig. $1 E, F$ ). Both NL1AB/NL2 and NL2A/NL1 massively enhanced EPSC (Fig. $1 D, E$ ). The latter result - which is to be expected since the parental NL1AB and NL2A constructs have similar effects on EPSC-demonstrates that the NL chimeras are expressed and functional. Together, the data indicate that the enhancement of IPSC is conferred by the extracellular domain of NL2 (Fig. 1F).

$\beta \mathrm{Nrxn} 1$ is prominently expressed in CA3 pyramidal neurons but only weakly in interneurons

The above experiments indicate that the extracellular domain of NL2, but not that of NL1AB, contains the determinant that enhances inhibitory synaptic transmission. Since the extracellular domain of NLs mediate selective interactions with Nrxns, we hypothesized that differential expression of specific Nrxn isoforms with distinct affinities for NL1 and NL2 in axons of GABAergic versus glutamatergic neurons underlies the differential effect of NL2 and NL1 on IPSC. As a first step to test this hypothesis, we performed singlecell qPCR and measured the expression of specific Nrxn isoforms in CA3 pyramidal neurons, the excitatory presynaptic partner of CA1 pyramidal neurons, as well as in various CA1 interneurons that provide inhibitory input to CA1 pyramidal cells. We harvested cytoplasm through wholecell patch pipettes and quantified the expression of $\beta \mathrm{Nrx} 1-3$ or $\alpha \mathrm{Nrxn} 1-3$ genes relative to GAPDH for each neuron. Parallel with qPCR of Nrxns, the pyramidal and interneuronal cell types were identified by conventional reverse transcription (RT)-PCR for cell-type markers (see Materials and Methods, Diagnostic PCR) and divided on this basis into three interneuron groups: parvalbumin (PV)-, somatostatin (Sst)-, and cholecystokinin (Cck)-expressing interneurons. Notably, the expression of $\beta \mathrm{Nrxn} 1$ in all three of these interneuron subtypes was much lower than that in CA3 pyramidal neurons (Fig. $3 A$ ). In contrast, there was no statistically significant differential expression of $\beta \mathrm{Nrxn} 2$ or $\beta \mathrm{Nrxn} 3$ between pyramidal neu-
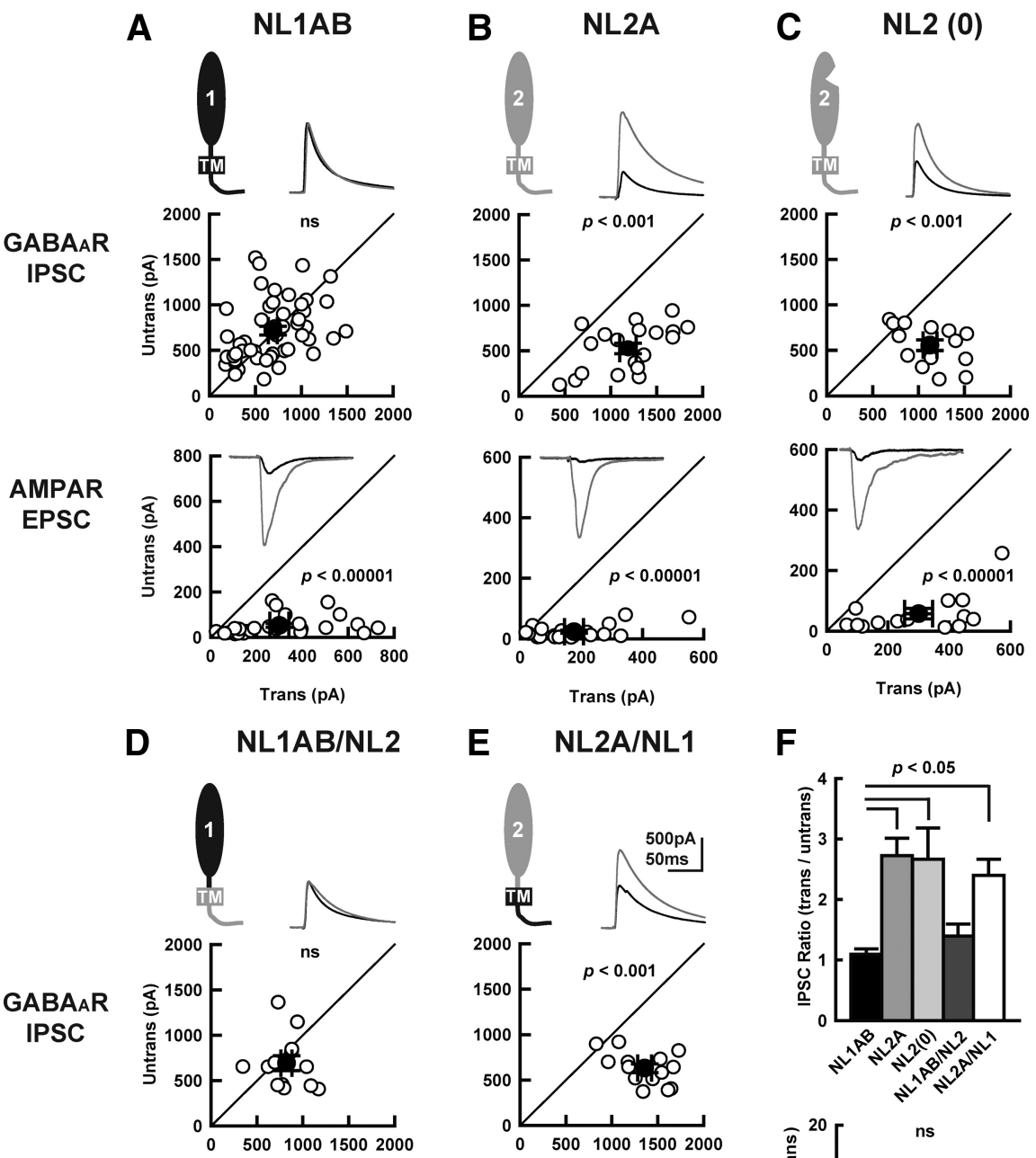

F
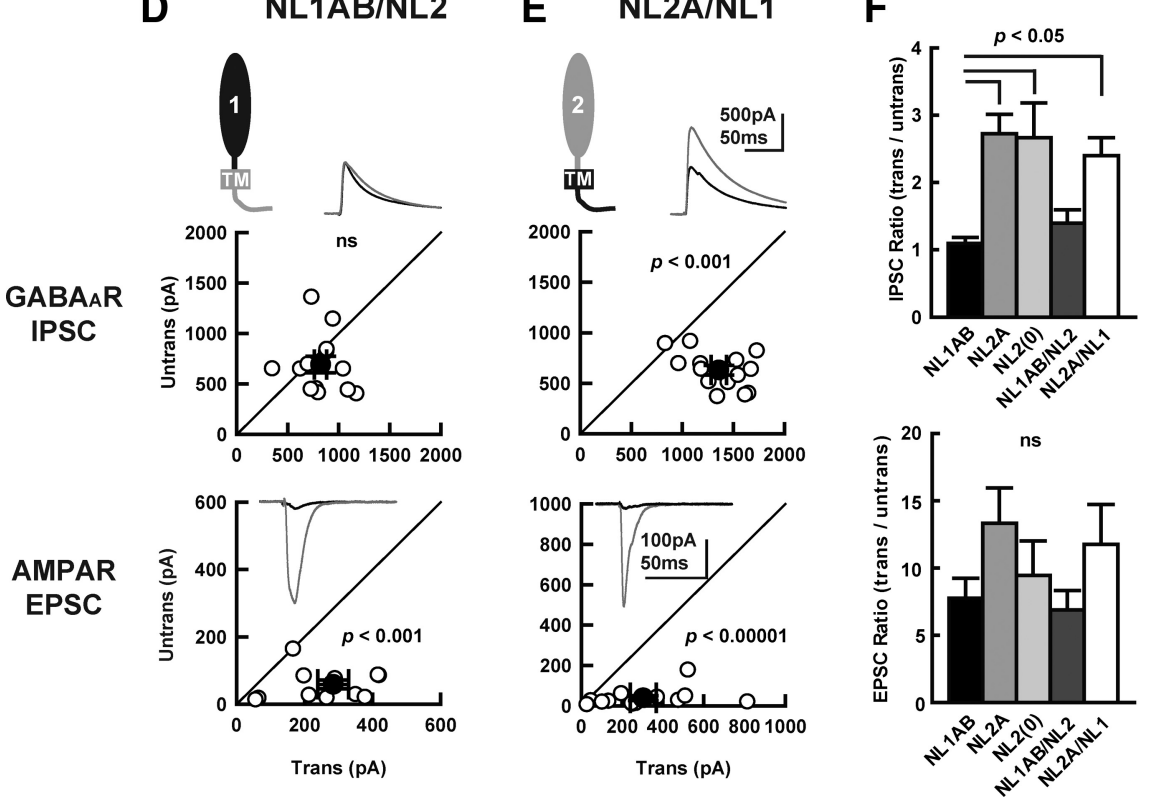

Figure 1. The extracellular domain of NL confers the synaptic specificity of NL isoforms. Effect of overexpression of NL isoforms and chimeric mutants on $\mathrm{GABA}_{\mathrm{A}} \mathrm{R}$-mediated IPSC and AMPAR-mediated EPSC in hippocampal CA1 pyramidal cells, respectively. $A-E$, IPSC (middle) and EPSC (bottom) amplitudes were plotted for each pair of transfected (Trans) and neighboring untransfected (Untrans) cells (open symbols). Filled symbols indicate the mean \pm SEM. Diagram of wild-type and chimera constructs of NL1 and NL2 (top, left). TM, transmembrane domain. Right and bottom inset, Superimposed sample IPSCs and EPSCs from pairs of transfected (gray) and untransfected (black) cells. Stimulus artifacts were truncated. NL1AB (A), NL2A (B), NL2(0) (C), NL1AB/NL2 (D), and NL2A/NL1 (E). Numbers of cell pairs: NL1 (GABA R-IPSCS/AMPAR-EPSCs: 49/25), NL2A (20/19), NL2(0) (15/14), NL1AB/NL2 $(13 / 12)$, and NL2A/NL1 (14/13). The number of tested slice cultures is the same as that of cell pairs. $\boldsymbol{F}$, Summary of effect of NL constructs on IPSCS (top) and EPSCS (bottom). Each bar represents the average of ratios obtained from multiple pairs of transfected and untransfected neighboring neurons. ns, not significant. Mann-Whitney test. Error bars indicate SEM (for all figures).

rons and interneuronal cells. This result suggests that the overall expression of $\beta \mathrm{Nrxn}$ isoforms in excitatory neurons is higher than that in interneurons. The expression of $\alpha \mathrm{Nrxn} 1$, but not of 2 and $\alpha \mathrm{Nrxn} 3$, was also elevated in CA3 pyramidal neurons compared with CA1 interneurons (Fig. 3B).

To evaluate the cell type-specific expression of Nrxns at the protein level, we next performed immunocytochemistry for $\beta$ Nrxn1 in presynaptic terminals of excitatory and inhibitory neurons. Using an anti- $\beta$ Nrxn 1 antibody that detected only 
A

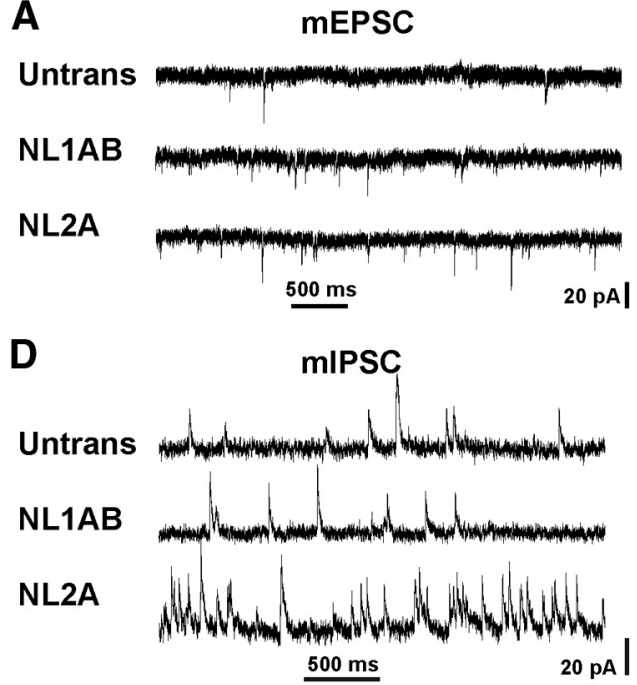

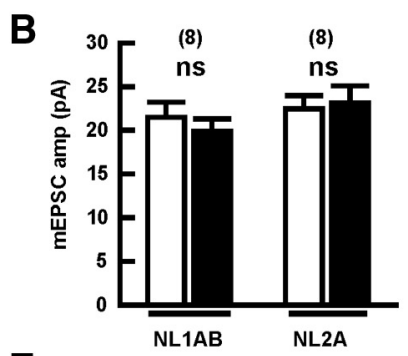

E

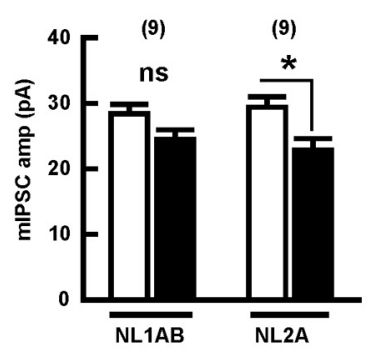

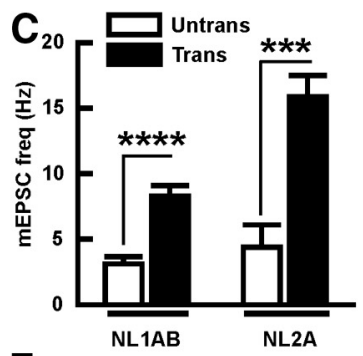

$\mathbf{F}$

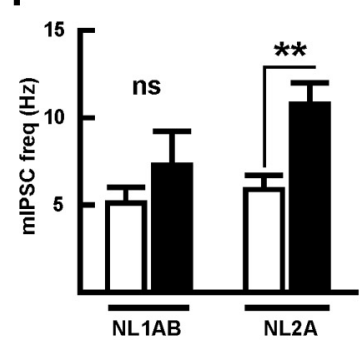

G

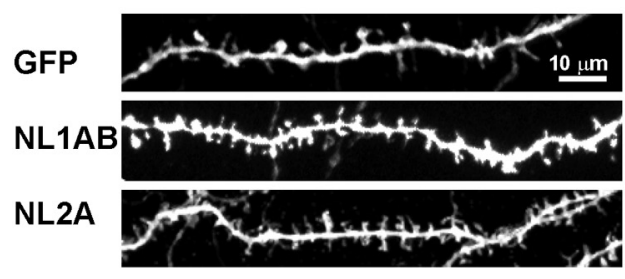

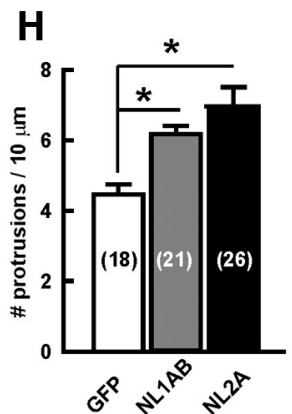

I

$\mathbf{J}$
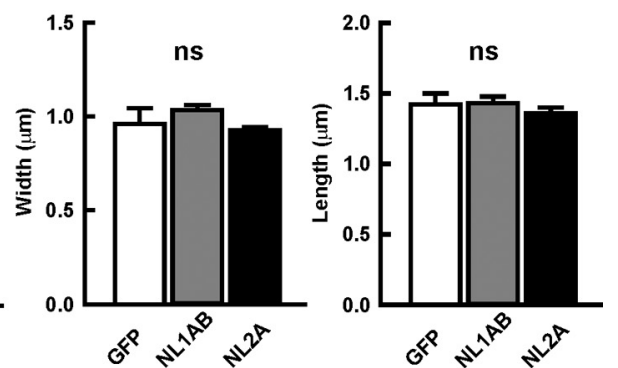

Figure 2. Effect of overexpression of NL isoforms on mPSCs and dendritic morphology. Effect of NL overexpression on AMPAR- $(\boldsymbol{A}-\boldsymbol{C})$ and $G A B A_{A} R-(D-F)$ mediated miniature synaptic transmission in cultured hippocampal CA1 pyramidal neurons. $A, D$, Sample traces from NLs transfected and neighboring untransfected (Untrans) neurons. Summary of the effect of NL isoforms on $\operatorname{AMPAR}-m E P S C S(B, C)$ and GABA $\mathrm{R}$-mIPSCS $(\boldsymbol{E}, \boldsymbol{F})$. Amplitude and frequency of miniature synaptic events of NL isoforms were compared with those of neighboring untransfected cells. Numbers of cell pairs were NL1AB (mIPSC, mEPSC): (9, 8); NL2A (9, 8). ns, not significant. ${ }^{*} p<0.05,{ }^{* *} p<0.01,{ }^{* * *} p<0.001$, ${ }^{* * * *} p<0.0001$, Student's $t$ test. G, Immunostaining for GFP in control cells expressing GFP (top), cells coexpressing GFP and NL1AB (middle), and GFP with NL2A (bottom). Effect of NL overexpression on average density $(\boldsymbol{H})$, head width $(\boldsymbol{I})$, and length $(\boldsymbol{J})$ of dendritic protrusions. Dendritic morphology was visualized by cotransfected GFP. Three days after transfection, neurons were fixed by PFA and immunostained for GFP. ${ }^{*} p<0.05$, Kruskal-Wallis ANOVA.

$\beta$ Nrxn 1 and its splice variants (Fig. $4 A$ ), we measured the area of $\beta$ Nrxn 1 puncta overlapping with the presynaptic markers of excitatory (VGluT1) or inhibitory (VGAT) synapses in cultured hippocampal neurons (Fig. $4 B$ ). Importantly, $\beta$ Nrxn1 colocalized largely with VGluT1: the area of $\beta$ Nrxn 1 puncta overlapping with VGluT1 was much higher than with VGAT, indicating a specific association of $\beta$ Nrxn 1 with excitatory synapses (colocalization of $\beta \mathrm{Nrxn} 1$ and VGluT1: $61.7 \pm 3.3 \% ; \beta \mathrm{Nrxn} 1$ and VGAT: $3.45 \pm 0.4 \%$; $\beta$ Nrxn1, VGluT1, and VGAT: $1.4 \pm 0.3 \%$; $n=14$ neurons, $>300$ puncta were measured from each neuron, $p<0.00001$, Student's $t$ test). The degree of colocalization of excitatory and inhibitory synapse markers VGluT1 and VGAT $(2.73 \pm 0.4 \%)$ was similar to that of $\beta \mathrm{Nrxn} 1$ and VGAT, supporting that $\beta \mathrm{Nrxn} 1$ is an excitatory synapse-specific Nrxn isoform. We also performed staining of mouse hippocampal dissociated neurons (DIV 21) and obtained similar results (colocalization of $\beta$ Nrxn 1 and VGluT1: $60.6 \pm 9.3 \% ; \beta$ Nrxn1 and VGAT: $16.1 \pm$ $11.3 \%$; $n=13$ neurons, $p<0.00001$, Student's $t$ test, data not shown). In addition, we confirmed the specific localization of $\beta \mathrm{Nrxn} 1$ in excitatory synaptic terminals in hippocampal slice culture (Fig. $4 C$ ) (colocalization of $\beta$ Nrxn1 and VGluT1: $56.5 \pm$ $1.8 \%, \beta$ Nrxn1 and VGAT: $1.4 \pm 0.11 \%, \beta$ Nrxn1, VGluT1, and VGAT: $0.6 \pm 0.08 \%, n=6$ slice cultures $/ 3$ rats, $>1500$ puncta were measured from each slice, $p<0.00001$, Student's $t$ test). Furthermore, we measured the expression of $\beta \mathrm{Nrxn}$ in axonal segments of excitatory and PV-expressing neurons. Axonal segments of excitatory neurons (marked by transfected BFP) were immunoreactive for anti- $\beta \mathrm{Nrxn} 1$ antibody (Fig. $4 D$ ). In contrast, the axonal projections of PV interneurons visualized by PV immunoreactivity displayed lack of $\beta \mathrm{Nrxn} 1$ signals (Fig. $4 E$ ). Together with the predominant expression of $\beta$ Nrxn 1 mRNA in excitatory neurons (Fig. 3 ), the above immunostaining results provide strong evidence that $\beta \mathrm{Nrxn} 1$ is predominantly localized in the presynaptic terminals of excitatory rather than inhibitory neurons.

NL1AB can induce functional inhibitory synaptic connections when $\beta$ Nrxn 1 is ectopically expressed in inhibitory interneurons

$\beta$ Nrxns bind directly to NL1, including the NL1AB isoform (Boucard et al., 2005; Comoletti et al., 2006, 2007; Ara ç et al., 2007; Chen et al., 2008; Reissner et al., 2008; Koehnke et al., 2010). Notably, the binding affinity of NL2 and $\beta$ Nrxn has been reported as $\sim 300$-fold lower than that of NL1- $\beta$ Nrxn (Comoletti et al., 2006; Leone et al., 2010, but also see Koehnke et al., 2010). We hypothesized that the specific ability of postsynaptic NL1AB overexpression to induce excitatory but not inhibitory synaptic transmission is due to the abundant expression of $\beta$ Nrxns, especially $\beta$ Nrxn 1 , in CA3 pyramidal neurons but the relatively low expression of $\beta$ Nrxns in inhibitory interneurons. 

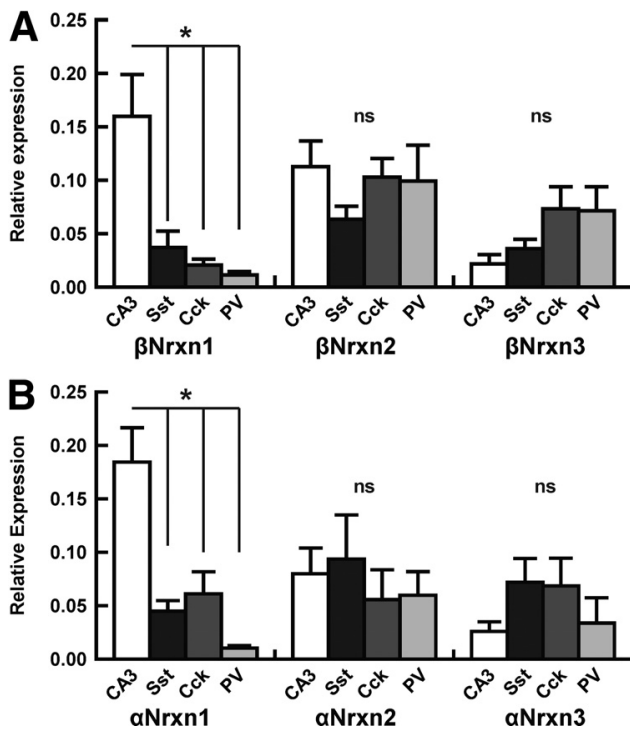

Figure 3. $\quad \beta N r x n 1$ is differentially expressed between excitatory and inhibitory neurons. Summary of single-cell gene expression of $\beta \operatorname{Nrxn}(\boldsymbol{A})$ and $\alpha \operatorname{Nrxn}(\boldsymbol{B})$ in hippocampal CA3 pyramidal neurons and CA1 interneurons. The quadruplex single-cell qPCR was performed in CA3 pyramidal neurons and CA1 interneurons. Interneuronal-types were divided into three groups: PV-, Sst-, and Cck-expressing interneurons. The expression level was normalized to that of GAPDH. Each bar represents the average expression of $\beta \operatorname{Nrxn}(\boldsymbol{A})$ and $\alpha \operatorname{Nrxn}(\boldsymbol{B})$ isoforms in CA3 pyramidal neurons and $\mathrm{CA} 1$ interneurons. Note that both $\alpha \mathrm{Nrxn} 1$ and $\beta \mathrm{Nrxn} 1$ were prominently expressed in CA3 pyramidal neurons compared with interneurons. The number in each bar represents the number of cells tested. Numbers of cells: $\beta \mathrm{Nrxn}[\mathrm{CA3}$ (18); $\mathrm{Sst}$ (25: includes 4 Cck, 5 Calb1, 1 Calb2, 1 Calb1/Calb2, 1 PV, and 1 PV/Cck/Calb1 coexpressing neurons); Cck (23: includes 2 VIP, 4 Sst, 1 PV, and 1 Sst/PV/Calb1 coexpressing neurons); PV (16: includes 1 Sst, 1 Cck, 1 Calb1, 1 Calb2, and 1 Sst/Cck/Calb1 coexpressing neurons)], $\alpha$ Nrxn [CA3 (8); Sst (7: includes 2 Calb1 and 1 VIP coexpressing neurons); (ck (5: includes 1 Calb1 coexpressing neurons); $\mathrm{PV}(4)$ ]. $p<0.001$ ( $\beta$ Nrxn) and $p=0.004(\alpha$ Nrxn) by one-way ANOVA, posthoc Dunnett test ${ }^{*} p<0.05$.

To test the hypothesis that the differential expression level of $\beta N r x n s$ in pyramidal versus inhibitory neurons accounts for the distinct effect that NL1AB and NL2A overexpression has on IPSC, we manipulated both the presynaptic and postsynaptic neurons and measured their functional connectivity using dual whole-cell recording of neuronal pairs. First, we confirmed that the effect of postsynaptic overexpression of NL isoforms in pyramidal neurons was reproduced on unitary inhibitory synaptic transmission (UIPSC). We performed simultaneous presynaptic and postsynaptic double whole-cell recording from a "postsynaptic" CA1 pyramidal neuron cotransfected with NL constructs and GFP and a visually identified "presynaptic" inhibitory interneuron located within $100 \mu \mathrm{m}$ of the transfected CA1 pyramidal neuron (Fig. 5A). Presynaptic release was evoked by a pair of depolarization pulses with a $50 \mathrm{~ms}$ interval in the untransfected interneuron while postsynaptic responses were measured from the CA1 pyramidal neuron transfected with GFP or with GFP and NL. As predicted from previous results (Fig. 1), the postsynaptic pyramidal neurons transfected with NL2A, but not NL1AB, showed significantly larger uIPSCs than neurons expressing GFP alone (Fig. 5B). In addition, NL2A-transfected pyramidal neurons had higher synaptic connectivity ( $\sim 80 \%$ vs $\sim 45 \%$ for GFP only) with nearby inhibitory interneurons, as scored by the fraction of neuron pairs found to be synaptically connected (Fig. 5C). We next tested the effect of presynaptic transfection of Nrxn1 isoforms on IPSCs recorded from untransfected pyramidal neurons. In this experiment, we had to manipulate the expression of Nrxn isoforms in the presynaptic interneurons. However, the low efficiency of the biolistic transfection was not the ideal transfection method for interneurons, which represent only $\sim 10 \%$ of all cells in hippocampus. We therefore used a single-cell electroporation technique to transfect Nrxns into CA1 interneurons (see Materials and Methods) (Haas et al., 2001). We transfected three Nrxn1 isoforms- $\beta \mathrm{Nrxn} 1$ containing an insertion at site 4 $[\beta \operatorname{Nrxn} 1(4+)]$, lacking splice insertion at site $4[\beta \operatorname{Nrxn}(4-)]$, or $\alpha \operatorname{Nrxn} 1(4-)$ - together with marker DsRed2 into visually identified CA1 interneurons. Two days after electroporation, we performed dual whole-cell recording from Nrxn1-transfected presynaptic interneurons (DsRed2 positive) and nearby (within $100 \mu \mathrm{m}$ ) untransfected postsynaptic pyramidal neurons (Fig. $5 D-F)$. Control cell pairs consisted of presynaptic interneurons transfected with DsRed2 alone and untransfected postsynaptic pyramidal neurons. In cell pairs overexpressing $\beta \operatorname{Nrxn} 1(4+$ or $4-)$ in presynaptic interneurons, there was no detectable change in uIPSC or connectivity (Fig. $5 E, F$ ). Similarly, presynaptic overexpression of $\alpha \operatorname{Nrxn1} 14-)$, a candidate binding partner for NL2 but not NL1AB (Boucard et al., 2005; Kang et al., 2008), did not enhance connectivity or uIPSC compared with control cell pairs. Thus neither overexpression of NL1 in the postsynaptic pyramidal cell (Figs. $1 A, 5 B, C$ ), nor overexpression of Nrxn alone in the presynaptic inhibitory interneuron (Fig. $5 E, F$ ), is sufficient to enhance the strength of connection between those cells.

We hypothesized that the sole overexpression of postsynaptic NL1AB or presynaptic Nrxn1 was not sufficient for induction of inhibitory synapses because the expression of the respective binding partners (presynaptic $\beta$ Nrxns or postsynaptic NLs) is limiting. This hypothesis predicts that concomitant overexpression of $\beta \mathrm{Nrxn}$ in presynaptic interneurons together with NL1AB in postsynaptic pyramidal neurons should result in enhanced inhibitory synaptic transmission between the two cells. Thus, we tested the effect of simultaneous transfection of Nrxn1 in the presynaptic interneuron and NL1AB in the postsynaptic pyramidal neuron (Fig. 6). The presynaptic and postsynaptic dual gene transfection was performed using a combination of biolistic gene gun (postsynaptic pyramidal neurons) and single-cell electroporation (presynaptic interneurons). Indeed, we observed an increase in connectivity in the cell pairs expressing $\beta \mathrm{Nrxn1} 14+$ or $4-)$ in the presynaptic interneuron and NL1AB in the postsynaptic pyramidal neuron. All dually transfected cell pairs (100\%, $23 / 23$ cell pairs tested) showed functional inhibitory synaptic connections, compared with $51.1 \%$ (48/94 pairs) for control cell pairs (Fig. $6 \mathrm{C}$ ). Moreover, the dually transfected cell pairs that expressed presynaptic $\beta \mathrm{Nrxn} 1(4-)$ and postsynaptic NL1AB exhibited markedly enhanced uIPSC amplitudes compared with control pairs (Fig. $6 B$ ). Cell pairs transfected with $\beta \operatorname{Nrxn1}(4+)$ and NL1AB showed a slight increase in mean uIPSC amplitude that did not reach statistical significance. Since postsynaptic transfection of NL1AB by itself did not alter inhibitory synaptic transmission (Figs. $1 A, 5 B$ ), these results indicate that the enhancement of connectivity and uIPSC is due to specific transsynaptic interaction of $\beta \mathrm{Nrxn} 1$ and NL1AB. The greater effect of $\beta \mathrm{Nrxn} 1(4-)$ correlates with its higher binding affinity for NL1 containing splice insertion at site B (Boucard et al., 2005; Reissner et al., 2008; Koehnke et al., 2010). Notably, presynaptic interneurons overexpressing $\alpha \operatorname{Nrxn1} 14-$ ), which does not bind to NL1AB (Boucard et al., 2005), did not increase uIPSC amplitude or connectivity with NL1AB-transfected pyramidal neurons, corroborating the importance of specific trans-synaptic Nrxn-NL interaction on functional synapse induction (Fig. $6 B, C$ ). 


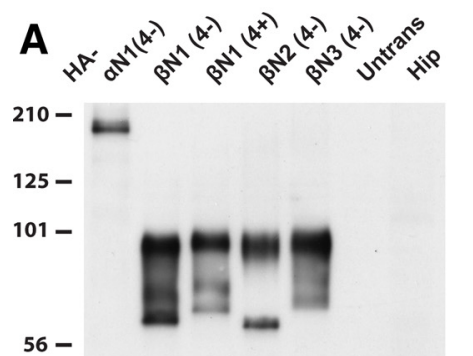

kD
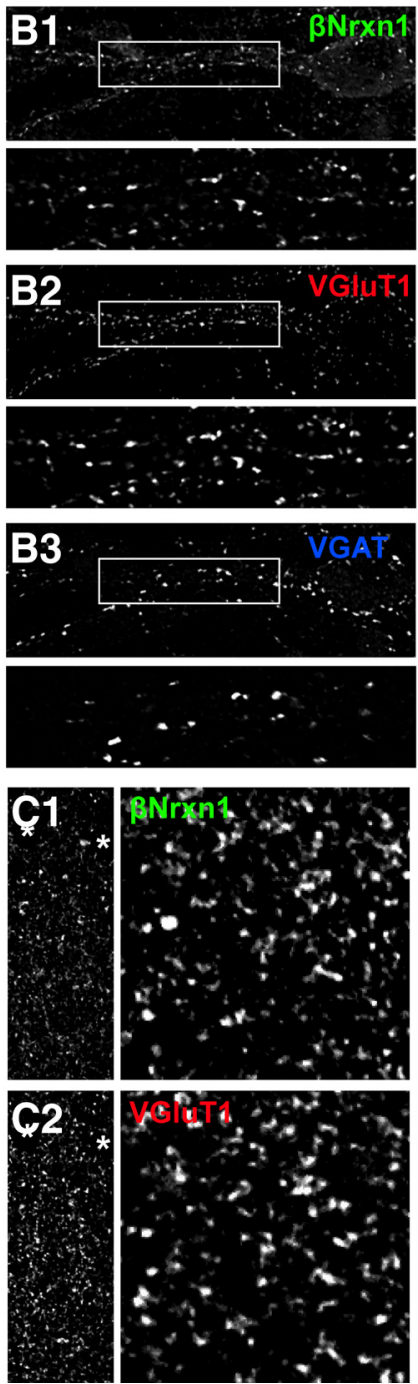
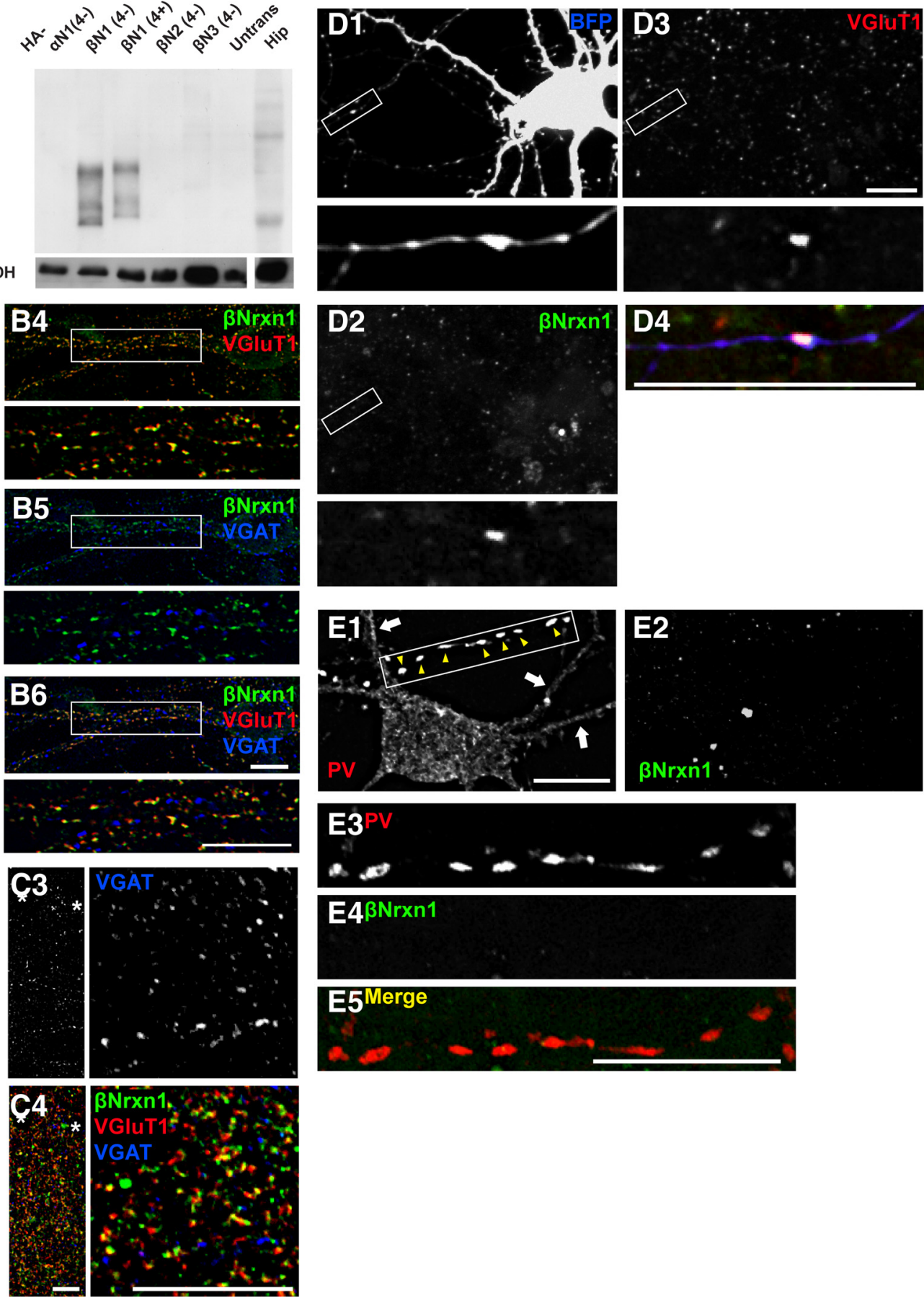

Figure 4. $\beta \mathrm{Nrxn} 1$ is predominantly expressed in excitatory presynaptic sites. $\boldsymbol{A}$, Specificity of $\beta \mathrm{Nrxn} 1$ antibody confirmed by probing lysates from HEK293T cells overexpressing HA-tagged $\alpha \operatorname{Nrxn1}(4-), \beta \operatorname{Nrxn1}(4 \pm)$, $\beta$ Nrxn 2(4-) or $\beta$ Nrxn3(4-), and hippocampal extract. Immunoblotting images with HA (left) or $\beta$ Nrxn1-specific (right) antibody. Membranes were first probed with rabbit HA antibody, stripped, then reprobed with mouse $\beta \mathrm{Nrxn} 1$ or GAPDH antibody. The expression of $\beta$ Nrxn1 isoform was confirmed in hippocampus. $B$, Cultured hippocampal neurons (DIV 14) were fixed and immunostained for $\beta \operatorname{Nrxn1}$ (B1, green in B4 and B5), VGluT1 (B2, red in B4 and B6), and VGAT (B3, blue in B5 and B6). Projection (up) or single-plane (bottom) confocal images were taken from dendritic segments. C, Organotypic hippocampal slice cultures (DIV 8) were fixed and immunostained for $\beta$ Nrxn1 (C1, green in (4), VGluT1 (C2, red in (4), and VGAT (C3, blue in C4). Left, Single-plane low-magnification confocal images were taken from hippocampal CA1 stratum pyramidale and radiatum areas. Right, Single-plane high-magnification images in hippocampal stratum radiatum area. Asterisks represent cell bodies in stratum pyramidale. $D$, Cultured rat hippocampal neurons (DIV 11) were transfected with BFP. Three days after transfection (DIV $11+3$ ), endogenous $\beta$ Nrxn1 and VGluT1 were immunostained with $\beta$ Nrxn1 (D2, green in D4) and VGluT1 (D3, red in D4) antibodies, and the axonal segment of transfected spiny excitatory neurons was visualized by BFP epifluorescence (D1, blue in D4). All VGluT1 puncta in axonal segments were colocalized with $\beta \mathrm{Nrxn1}$ ( $n=4$ neurons, $>20 \mathrm{VGluT1}$ puncta were tested in each neurons). $\boldsymbol{E}$, Lack of $\beta \mathrm{Nrxn} 1$ immunoreactivity in axonal segment of PV + interneurons. Fixed cultured hippocampal neurons (DIV 14) were immunostained for PV (E1, E3, red in E5) and $\beta$ Nrxn1 (E2, E4, green in E5). E1, E2, Low-magnification images of PV-expressing neuron. Arrows, dendritic segments; yellow arrowheads, axonal terminals. E3-E5, High-magnification images of region in white rectangle in E1. Scale bars: $10 \mu \mathrm{m}$. 
NL2A couples with $\alpha$ Nrxn 1 to form functional inhibitory synapses

Biochemical assays revealed that NL2 directly binds to $\alpha \mathrm{Nrxn} 1$ (Boucard et al., 2005). Interestingly, the binding affinity of NL2 and $\beta$ Nrxn appears to be much lower than that of NL1- $\beta$ Nrxn (Comoletti et al., 2006; Leone et al., 2010), but also see Koehnke et al. (Koehnke et al., 2010). However, the coculture studies suggested that overexpression of either $\beta$ Nrxns or $\alpha$ Nrxns triggers the assembly of inhibitory postsynaptic proteins (Nam and Chen, 2005; Chih et al., 2006; Kang et al., 2008). To address in neurons which Nrxn isoform synergizes with NL2A to form functional inhibitory synapses, we next tested the cell pairs that overexpressed $\alpha$ Nrxns or $\beta$ Nrxns in the presynaptic interneurons and NL2A in the postsynaptic neurons. The cell pairs transfected with $\alpha \operatorname{Nrxn} 1(4-)$ and NL2A in presynaptic and postsynaptic neurons, respectively, showed $100 \%$ synaptic connectivity (19/19 cell pairs tested) as well as increased uIPSC amplitude, compared with control or NL2A postsynaptically transfected cell pairs (Fig. 6D-F). Presynaptic transfection of $\beta \mathrm{Nrxn} 1(4-)$ or $\beta \operatorname{Nrxn} 1(4+)$ did not further strengthen inhibitory synaptic transmission with postsynaptically transfected NL2A; rather, it lowered the UIPSC amplitude and connectivity to a level not significantly different from control cell pairs (Fig. 6E,F). This suggests that the effect of postsynaptic NL2A transfection on inhibitory synaptic transmission was neutralized by presynaptically overexpressed $\beta \mathrm{Nrxn1}(4-)$ or $\beta \mathrm{Nrxn} 1$ $(4+)$. This may indicate that presynaptically transfected $\beta \mathrm{Nrxn} 1(4-)$ or $\beta \mathrm{Nrxn1}(4+)$ acts as a negative regulator for inhibitory synaptic function by disrupting the protein interaction of endogenous $\alpha$ Nrxns with NL2. In these presynaptic and postsynaptic dual recordings (Figs. 5, 6), the tested interneurons were "typed" by diagnostic PCR of markers (PV, Sst, etc.), and there was no interneuronal type-specific effect of Nrxns and NLs on connectivity and UIPSC amplitude (data not shown).

\section{Discussion}

Despite the lines of evidence suggesting that different combinations of trans-synaptic adhesion molecules can determine either excitatory or inhibitory synapse function, the specifics of this model have not been elucidated. NL1AB and NL2A have been contrasted as the excitatory and inhibitory synapse-specific postsynaptic adhesion molecules, respectively; however, the molecular mechanisms that confer specific function to NL1 and NL2 have not been fully understood. Our results demonstrate that the extracellular domain of these NL isoforms confers their differential function on GABAergic synaptic transmission. We also found that $\beta \mathrm{Nrxn} 1$ is specifically local-

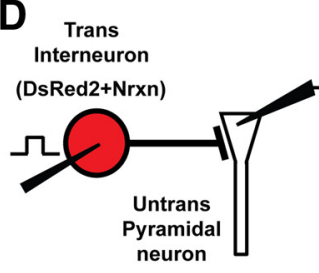

pre: $\beta$ Nrxn1(4-)

$\alpha$ Nrxn1(4-)
Untrans
B C

E

$\mathbf{F}$
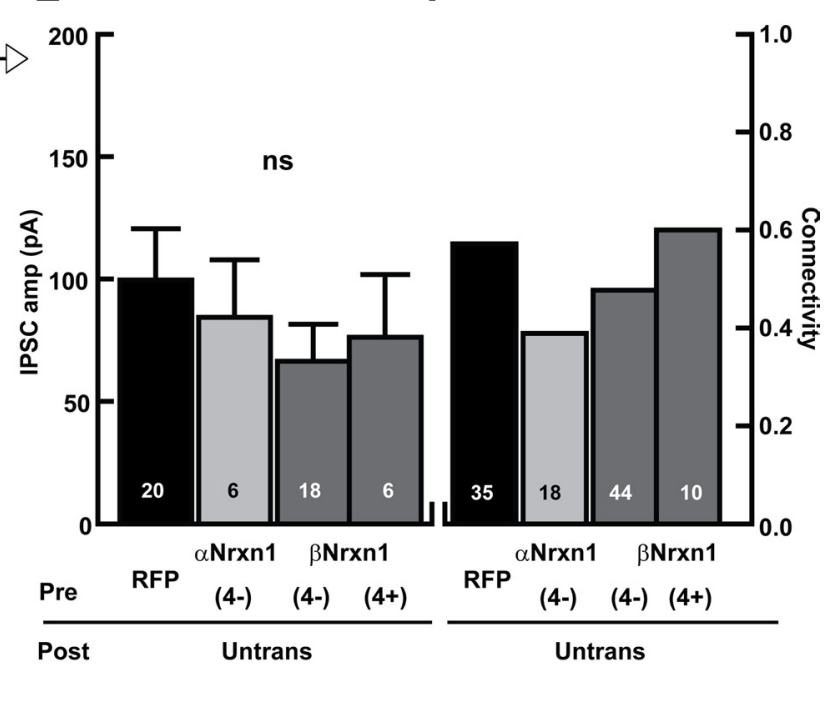

RFP

(4-) (4-) (4+)

Untrans

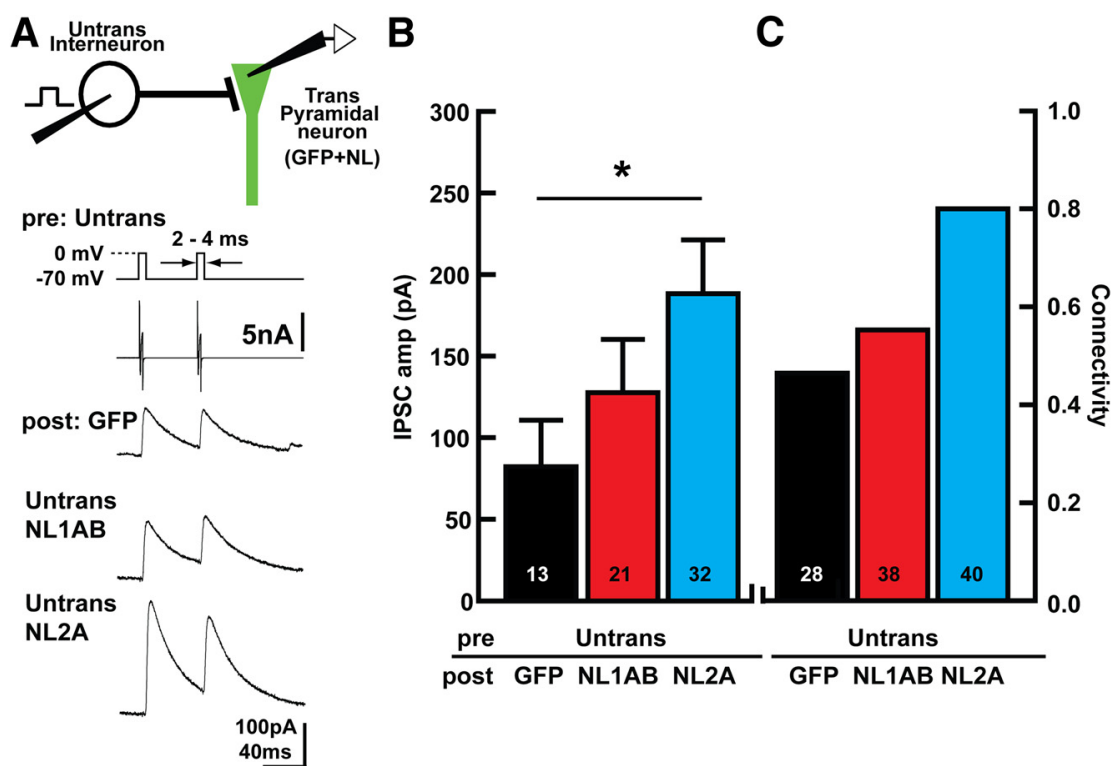

Figure 5. Presynaptic overexpression of Nrxn alone does not change inhibitory synaptic transmission. $\boldsymbol{A}-\boldsymbol{C}$, Effect of postsynaptic overexpression of NLS (NL1, red; NL2: blue) on unitary inhibitory synaptic transmission in hippocampal CA1 pyramidal neurons. $\boldsymbol{A}$, Configuration of dual whole-cell recording (top) and averaged sample uIPSC traces by two presynaptically applied depolarization commands (bottom). $\boldsymbol{B}, \boldsymbol{C}$, Summary of uIPSC amplitude (B) and connectivity (C). The cell pairs, which consist of untransfected interneurons and GFP-expressing postsynaptic pyramidal neurons, were used as the control. $p=0.014$ by one-way ANOVA, post hoc Dunnett test ${ }^{*} p<0.05$. D-F, Effect of presynaptic overexpression of Nrxns ( $\alpha$ Nrxn1, light gray; $\beta$ Nrxn1, dark gray) on inhibitory synaptic transmission in hippocampal CA1 pyramidal neurons. $\boldsymbol{D}$, Configuration of dual whole-cell recording (top), and averaged sample uIPSC traces (bottom). Nrxns were overexpressed in CA1 interneurons by electroporation and postsynaptic response was measured from neighboring untransfected CA1 pyramidal neurons. Summary of uIPSC amplitude $(\boldsymbol{E})$ and connectivity $(\boldsymbol{F})$. Controls are neuronal pairs expressing DsRed2 presynaptically. Number in each bar represents the number of synaptically connected cell pairs $(\boldsymbol{B}, \boldsymbol{E})$ and total number of cell pairs tested $(\boldsymbol{C}, \boldsymbol{F})$.

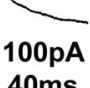

ized in excitatory rather than inhibitory presynaptic terminals. Using a dual presynaptic and postsynaptic gene transfection and recording technique, we demonstrated that the NL2A-mediated enhancement of inhibitory synaptic transmission can be assumed by NL1AB, an excitatory synapse-specific adhesion molecule, but only when an NL1AB binding partner ( $\beta$ Nrxn1, normally at low levels) is overexpressed in presynaptic interneurons. This indicates that the NL-Nrxn-mediated trans-synaptic interaction is sufficient to promote functional inhibitory synapses and implies that the inefficiency of NL1AB on inhibitory synaptic function is due to the physiologic absence of the primary 
A
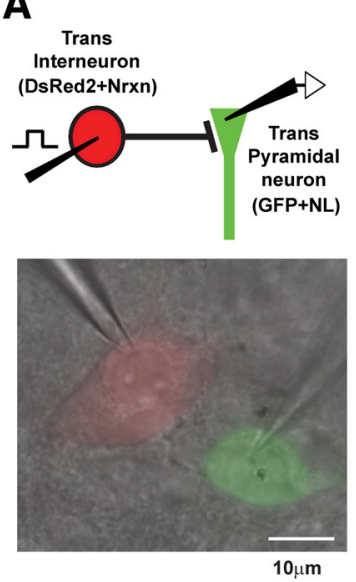

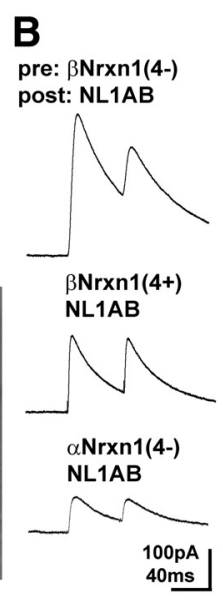

E

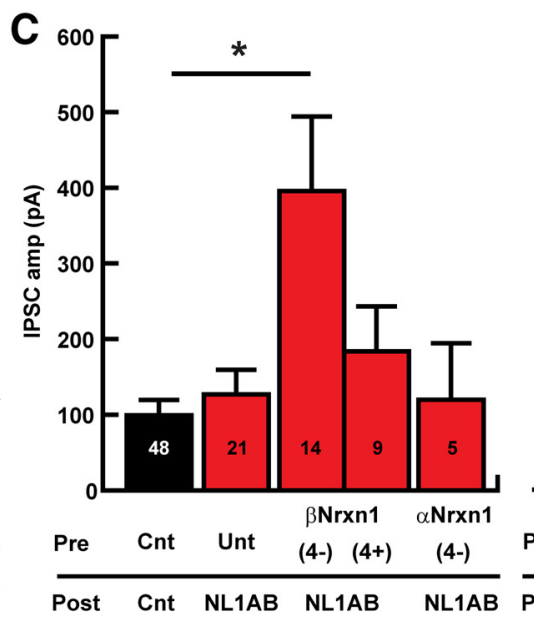

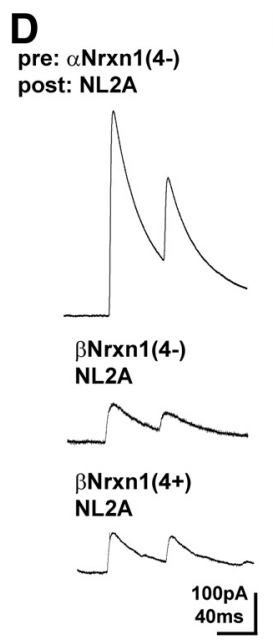

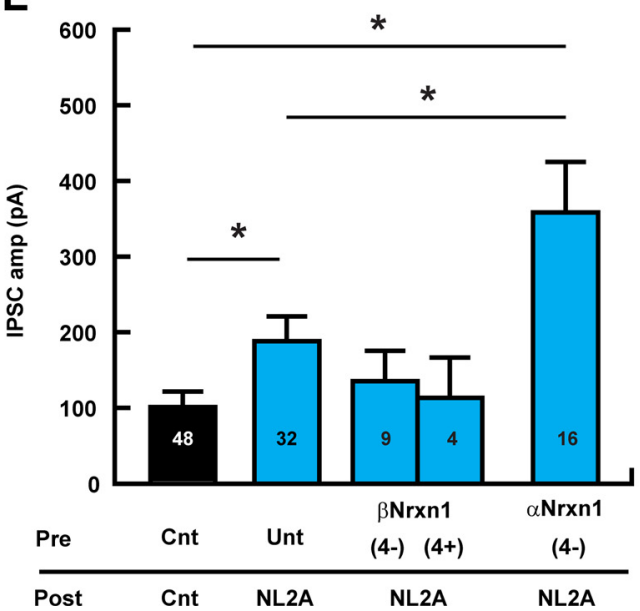

$\mathbf{F}$

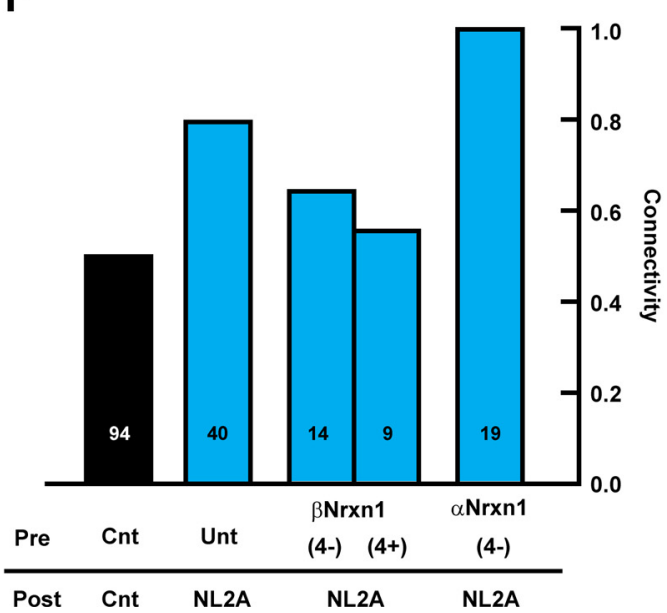

Figure 6. NL-Nrxn interaction confers the functional inhibitory synapse formation. $\boldsymbol{A}-\boldsymbol{C}$, Effect of dual overexpression of NL1AB (red) and $\beta$ Nrxn1 or $\alpha$ Nrxn1 in postsynaptic and presynaptic neurons on unitary inhibitory synaptic transmission. $A$, Configuration of dual whole-cell recording (left top), superimposed fluorescent and Nomarski image (left bottom), and averaged sample ulPSC traces (right). NLs were biolistically transfected in CA1 pyramidal neurons (green), and interneurons, which located within $100 \mu \mathrm{m}$ from NL-transfected neurons, were transfected Nrxns by electroporation (red). $\boldsymbol{B}, \boldsymbol{C}$, Summary of uIPSC amplitude $(\boldsymbol{B})$ and connectivity (C). $p=0.002$ by one-way ANOVA, post hoc Dunnett test ${ }^{*} p<0.05$. D-F, Effect of dual overexpression of NL2A (blue) and $\beta \mathrm{Nrxn} 1$ or $\alpha \mathrm{Nrxn} 1$ in postsynaptic and presynaptic neurons on unitary inhibitory synaptic transmission. $\boldsymbol{D}$, Averaged sample ulPSC traces. Summary of ulPSC amplitude $(\boldsymbol{E})$ and connectivity $(\boldsymbol{F})$. $p=0.00006$ by one-way ANOVA, post hoc Dunnett test ${ }^{*} p<0.05$. The bar graphs of postsynaptic overexpression of NL1AB and NL2A on ulPSC and connectivity in Figure 5,B and $C$, are included for a more comprehensive presentation of the finding in the main text. Thirty-one cell pairs expressing GFP and DsRed2 in postsynaptic and presynaptic neurons, and control pairs in Figure 5 were pooled as the control cell pairs (Cntl, black bars).

NL1AB binding partner, i.e., $\beta$ Nrxn1, in presynaptic interneurons.

The binding affinities between the various NL isoforms and Nrxn splice variants remain to be fully worked out. With regard to NL1, Boucard et al. (2005) reported that the NL1 splice variants containing a $\mathrm{B}$ insertion specifically bind to $\beta \operatorname{Nrxn} 1(4-)$, but not to $\beta \operatorname{Nrxn} 1(4+)$ or $\alpha \operatorname{Nrxn1}(4-)$. However, another study indicated that NL1AB is capable of binding to the LNS6 domains of $\alpha \operatorname{Nrxn} 1$ and $\beta \operatorname{Nrxn} 1(4+)$, albeit with lower affinity (Reissner et al., 2008; Koehnke et al., 2010). It has been reported that the binding affinity of $\beta \operatorname{Nrxn1}(4-)$ for the extracellular domain of NL2(0), which exhibited similar synaptic function as NL2A (Fig. 1C), is orders of magnitude lower than for NL1 $\Delta \mathrm{AB}$ (without splice insert $\mathrm{A}$ and $\mathrm{B}$ ) $\left[\mathrm{NL} 1 \Delta \mathrm{AB} / \beta \mathrm{Nrxn} 1(4-): K_{\mathrm{D}} \sim 29\right.$ $\mathrm{nM}, \mathrm{NL} 2 \Delta \mathrm{A} / \beta \mathrm{Nrxn} 1(4-)$ : $\sim 8.8 \mu \mathrm{M}$ ] (Comoletti et al., 2006), and this difference is attributed to the structural difference between the extracellular domain of NL1 and NL2 (Leone et al., 2010). However, another study (Koehnke et al., 2010) showed a similar affinity of NL1s and NL2s for $\beta$ Nrxn 1 s [e.g., $\beta \mathrm{Nrxn} 1(4-) / \mathrm{NL} 1 \mathrm{AB}: K_{\mathrm{D}}=1.21 \mu \mathrm{M}, \beta \mathrm{Nrxn} 1(4-) / \mathrm{NL} 2 \mathrm{~A}$ : $0.43 \mu \mathrm{M}]$.
Our experiment did not directly measure the biochemical binding affinity between NLs and Nrxns, but we presume that cell-pair connectivity and the amplitude of uIPSCs is a reflection of the extent of Nrxn-NL interaction between the two neurons of the pair. We found that cell pairs expressing $\beta \operatorname{Nrxn}(4+)$ or $\beta \mathrm{Nrxn}(4-)$ in interneurons and NL1AB in pyramidal neurons showed enhanced (100\%) synaptic connectivity, although the increased amplitude of uIPSC only reached statistical significance in $\beta \mathrm{Nrxn} 1(4-)$ and NL1AB overexpressing cell pairs (Fig. $6 A-$ $C)$. These results suggest that NL1AB can bind to both isoforms of $\beta$ Nrxn1 in the cell-cell context, and that the difference of uIPSC amplitude (perhaps a more quantitative measure) may be due to a stronger binding affinity of $\beta \operatorname{Nrxn1}(4-)$ for NL1AB (Reissner et al., 2008; Koehnke et al., 2010). In addition, cell pairs expressing $\alpha \operatorname{Nrxn}(4-)$ in interneurons and NL1AB in pyramidal neurons exhibited no significant synaptic effect compared with controls, consistent with the low binding affinity of NL1AB to $\alpha \operatorname{Nrxn}(4-)$ (Boucard et al., 2005).

Our connectivity study also suggests that the endogenous presynaptic binding partner of NL2 is $\alpha$ Nrxns, rather than $\beta$ Nrxns, because presynaptically overexpressed $\beta \mathrm{Nrxn} 1(4-)$ or $\beta \mathrm{Nrxn} 1$ 
$(4+)$ did not functionally interact with postsynaptically expressed NL2A to enhance inhibitory synapses, even though they were effective with postsynaptic NL1AB (Figs. 5, 6). Our qPCR result indicates that $\alpha \mathrm{Nrxn} 1$ is weakly expressed in PV + interneurons (Fig. 3). This may suggest that other $\alpha \mathrm{Nrxn}$ isoforms ( $\alpha$ Nrxn2 and $\alpha$ Nrxn3) work as the partner of NL2A to form functional inhibitory synapses in PV + interneurons (Kang et al., 2008). Multiple studies have reported that $\beta$ Nrxns are capable of inducing the clustering of inhibitory postsynaptic molecules (Chih et al., 2006; Kang et al., 2008). Perhaps NL3 could mediate the assembly of inhibitory postsynaptic molecules through an interaction with $\beta$ Nrxns (Budreck and Scheiffele, 2007). Further study using our dual cell transfection technique should help to reveal the functional consequences of not only additional specific NL-Nrxn interactions, but also any other trans-synaptic interactions.

Even though NLs are not absolutely essential for "structural" synapse formation in mouse knock-out models (Varoqueaux et al., 2006), it has been proposed that NLs validate the function (excitatory vs inhibitory) of the synapse because of their differential subcellular localizations. Since NL1 is usually associated with excitatory postsynaptic proteins (such as PSD-95), how does postsynaptic NL1AB cooperate with $\beta \mathrm{Nrxn} 1$ in presynaptic interneurons to assemble functional inhibitory synapses? Our results uncover the capability of NL1AB as an "organizer" of inhibitory synapses when there is a binding partner of NL1AB, $\beta \mathrm{Nrxn1}$, present in the presynaptic interneuron. It is reasonable to speculate that the intracellular domain of NL1 can serve as the adaptor domain for the assembly of inhibitory postsynaptic proteins. Although the intracellular domain of NL1 and NL2 are overall only moderately homologous ( $50 \%$ homology, $38 \%$ identity in amino acid sequence), the three characterized domains, the PDZ-binding motif, gephyrin-binding motifs, and critical domain (structurally unsolved), are highly conserved between NL1 and NL2 (Meyer et al., 2004; Poulopoulos et al., 2009; Shipman et al., 2011). Therefore, we propose that the transsynaptic interaction alone can nucleate functional inhibitory synapse formation, at least under overexpression conditions, and that any NL intracellular domain can perform the protein interactions required for the kind of synapse specified. Indeed, NL2 can bind to PSD-95 (Meyer et al., 2004) and colocalize with PSD-95 both in vitro (Levinson et al., 2005) and in vivo (Hines et al., 2008).

Our study sheds light on the hierarchical interaction of NLNrxn binding. Postsynaptic overexpression of NL2A by itself strongly increased both stimulation-evoked and unitary inhibitory synaptic transmission (Figs. $1 B, 5 B, C$ ). However, presynaptic transfection of any Nrxn1 isoforms had no effect on inhibitory synaptic function in untransfected postsynaptic neurons (Fig. $5 E, F)$. It is notable that the overexpression of $\alpha \operatorname{Nrxn} 1(4-)$, one of the strongest candidates for inhibitory presynaptic Nrxns (Chih et al., 2006; Kang et al., 2008), did not further promote functional inhibitory synapse formation on to untransfected pyramidal neurons. A similar result was observed for excitatory synapses (Futai et al., 2007). Presynaptic overexpression of $\beta \mathrm{Nrxn} 1(4-)$ in CA3 pyramidal neurons neither increased unitary excitatory synaptic transmission nor connectivity to neighboring untransfected pyramidal neurons, which might be because the number of endogenous postsynaptic NLs is limiting. This explanation is supported by our presynaptic and postsynaptic overexpression study because Nrxn1-overexpressing interneurons showed $100 \%$ of connectivity when the corresponding Nrxn binding partner was simultaneously transfected in postsynaptic pyramidal neurons (Fig. 6).
A large number of genetic studies has revealed the association of Nrxn1 with autism spectrum disorder, schizophrenia, mental retardation, and nicotine dependence (Reichelt et al., 2012). Furthermore, a loss-of-function mutation of NL2 was found in schizophrenia (Sun et al., 2011). Therefore, it is of particular interest to understand the trans-synaptic interaction between Nrxn 1 and NL2 on inhibitory synaptic function for better understanding of the synaptic pathophysiology of psychiatric illness.

\section{References}

Araç D, Boucard AA, Ozkan E, Strop P, Newell E, Südhof TC, Brunger AT (2007) Structures of neuroligin-1 and the neuroligin-1/neurexin- 1 beta complex reveal specific protein-protein and protein-Ca2+ interactions. Neuron 56:992-1003. CrossRef Medline

Barrow SL, Constable JR, Clark E, El-Sabeawy F, McAllister AK, Washbourne P (2009) Neuroligin 1: a cell adhesion molecule that recruits PSD-95 and NMDA receptors by distinct mechanisms during synaptogenesis. Neural Dev 4:17. CrossRef Medline

Blundell J, Blaiss CA, Etherton MR, Espinosa F, Tabuchi K, Walz C, Bolliger MF, Südhof TC, Powell CM (2010) Neuroligin-1 deletion results in impaired spatial memory and increased repetitive behavior. J Neurosci 30: 2115-2129. CrossRef Medline

Boucard AA, Chubykin AA, Comoletti D, Taylor P, Südhof TC (2005) A splice code for trans-synaptic cell adhesion mediated by binding of neuroligin 1 to alpha- and beta-neurexins. Neuron 48:229-236. CrossRef Medline

Brewer GJ, Torricelli JR, Evege EK, Price PJ (1993) Optimized survival of hippocampal neurons in B27-supplemented Neurobasal, a new serumfree medium combination. J Neurosci Res 35:567-576. CrossRef Medline

Budreck EC, Scheiffele P (2007) Neuroligin-3 is a neuronal adhesion protein at GABAergic and glutamatergic synapses. Eur J Neurosci 26:17381748. CrossRef Medline

Carlin RK, Grab DJ, Cohen RS, Siekevitz P (1980) Isolation and characterization of postsynaptic densities from various brain regions: enrichment of different types of postsynaptic densities. J Cell Biol 86:831-845. CrossRef Medline

Chen X, Liu H, Shim AH, Focia PJ, He X (2008) Structural basis for synaptic adhesion mediated by neuroligin-neurexin interactions. Nat Struct Mol Biol 15:50-56. CrossRef Medline

Chih B, Engelman H, Scheiffele P (2005) Control of excitatory and inhibitory synapse formation by neuroligins. Science 307:1324-1328. CrossRef Medline

Chih B, Gollan L, Scheiffele P (2006) Alternative splicing controls selective trans-synaptic interactions of the neuroligin-neurexin complex. Neuron 51:171-178. CrossRef Medline

Chubykin AA, Atasoy D, Etherton MR, Brose N, Kavalali ET, Gibson JR, Südhof TC (2007) Activity-dependent validation of excitatory versus inhibitory synapses by neuroligin-1 versus neuroligin-2. Neuron 54: 919-931. CrossRef Medline

Comoletti D, Flynn RE, Boucard AA, Demeler B, Schirf V, Shi J, Jennings LL, Newlin HR, Südhof TC, Taylor P (2006) Gene selection, alternative splicing, and post-translational processing regulate neuroligin selectivity for beta-neurexins. Biochemistry 45:12816-12827. CrossRef Medline

Comoletti D, Grishaev A, Whitten AE, Tsigelny I, Taylor P, Trewhella J (2007) Synaptic arrangement of the neuroligin/beta-neurexin complex revealed by X-ray and neutron scattering. Structure 15:693-705. CrossRef Medline

Futai K, Kim MJ, Hashikawa T, Scheiffele P, Sheng M, Hayashi Y (2007) Retrograde modulation of presynaptic release probability through signaling mediated by PSD-95-neuroligin. Nat Neurosci 10:186-195. CrossRef Medline

Gibson JR, Huber KM, Südhof TC (2009) Neuroligin-2 deletion selectively decreases inhibitory synaptic transmission originating from fast-spiking but not from somatostatin-positive interneurons. J Neurosci 29:1388313897. CrossRef Medline

Graf ER, Zhang X, Jin SX, Linhoff MW, Craig AM (2004) Neurexins induce differentiation of GABA and glutamate postsynaptic specializations via neuroligins. Cell 119:1013-1026. CrossRef Medline

Haas K, Sin WC, Javaherian A, Li Z, Cline HT (2001) Single-cell electroporation for gene transfer in vivo. Neuron 29:583-591. CrossRef Medline

Hines RM, Wu L, Hines DJ, Steenland H, Mansour S, Dahlhaus R, Singaraja 
RR, Cao X, Sammler E, Hormuzdi SG, Zhuo M, El-Husseini A (2008) Synaptic imbalance, stereotypies, and impaired social interactions in mice with altered neuroligin 2 expression. J Neurosci 28:6055-6067. CrossRef Medline

Jonas JC, Sharma A, Hasenkamp W, Ilkova H, Patan è G, Laybutt R, BonnerWeir S, Weir GC (1999) Chronic hyperglycemia triggers loss of pancreatic beta cell differentiation in an animal model of diabetes. J Biol Chem 274:14112-14121. CrossRef Medline

Kang Y, Zhang X, Dobie F, Wu H, Craig AM (2008) Induction of GABAergic postsynaptic differentiation by alpha-neurexins. J Biol Chem 283:2323-2334. Medline

Koehnke J, Katsamba PS, Ahlsen G, Bahna F, Vendome J, Honig B, Shapiro L, Jin X (2010) Splice form dependence of beta-neurexin/neuroligin binding interactions. Neuron 67:61-74. CrossRef Medline

Leone P, Comoletti D, Ferracci G, Conrod S, Garcia SU, Taylor P, Bourne Y, Marchot P (2010) Structural insights into the exquisite selectivity of neurexin/neuroligin synaptic interactions. EMBO J 29:2461-2471. CrossRef Medline

Levinson JN, Chéry N, Huang K, Wong TP, Gerrow K, Kang R, Prange O, Wang YT, El-Husseini A (2005) Neuroligins mediate excitatory and inhibitory synapse formation: involvement of PSD-95 and neurexin-1beta in neuroligin-induced synaptic specificity. J Biol Chem 280:17312-17319. CrossRef Medline

McAllister AK (2000) Biolistic transfection of neurons. Sci STKE 2000:pl1. Medline

Meyer G, Varoqueaux F, Neeb A, Oschlies M, Brose N (2004) The complexity of PDZ domain-mediated interactions at glutamatergic synapses: a case study on neuroligin. Neuropharmacology 47:724-733. CrossRef Medline

Missler M, Zhang W, Rohlmann A, Kattenstroth G, Hammer RE, Gottmann K, Südhof TC (2003) Alpha-neurexins couple Ca2 + channels to synaptic vesicle exocytosis. Nature 423:939-948. CrossRef Medline

Nakagawa T, Futai K, Lashuel HA, Lo I, Okamoto K, Walz T, Hayashi Y, Sheng M (2004) Quaternary structure, protein dynamics, and synaptic function of SAP97 controlled by L27 domain interactions. Neuron 44:453-467. CrossRef Medline

Nam CI, Chen L (2005) Postsynaptic assembly induced by neurexinneuroligin interaction and neurotransmitter. Proc Natl Acad Sci U S A 102:6137-6142. CrossRef Medline

Poulopoulos A, Aramuni G, Meyer G, Soykan T, Hoon M, Papadopoulos T,
Zhang M, Paarmann I, Fuchs C, Harvey K, Jedlicka P, Schwarzacher SW, Betz H, Harvey RJ, Brose N, Zhang W, Varoqueaux F (2009) Neuroligin 2 drives postsynaptic assembly at perisomatic inhibitory synapses through gephyrin and collybistin. Neuron 63:628-642. CrossRef Medline

Reichelt AC, Rodgers RJ, Clapcote SJ (2012) The role of neurexins in schizophrenia and autistic spectrum disorder. Neuropharmacology 62:1519-1526. CrossRef Medline

Reissner C, Klose M, Fairless R, Missler M (2008) Mutational analysis of the neurexin/neuroligin complex reveals essential and regulatory components. Proc Natl Acad Sci U S A 105:15124-15129. CrossRef Medline

Scheiffele P, Fan J, Choih J, Fetter R, Serafini T (2000) Neuroligin expressed in nonneuronal cells triggers presynaptic development in contacting axons. Cell 101:657-669. CrossRef Medline

Shipman SL, Schnell E, Hirai T, Chen BS, Roche KW, Nicoll RA (2011) Functional dependence of neuroligin on a new non-PDZ intracellular domain. Nat Neurosci 14:718-726. CrossRef Medline

Song JY, Ichtchenko K, Südhof TC, Brose N (1999) Neuroligin 1 is a postsynaptic cell-adhesion molecule of excitatory synapses. Proc Natl Acad Sci U S A 96:1100-1105. CrossRef Medline

Südhof TC (2008) Neuroligins and neurexins link synaptic function to cognitive disease. Nature 455:903-911. CrossRef Medline

Sun C, Cheng MC, Qin R, Liao DL, Chen TT, Koong FJ, Chen G, Chen CH (2011) Identification and functional characterization of rare mutations of the neuroligin-2 gene (NLGN2) associated with schizophrenia. Hum Mol Genet 20:3042-3051. CrossRef Medline

Ullrich B, Ushkaryov YA, Südhof TC (1995) Cartography of neurexins: $>1000$ isoforms generated by alternative splicing and expressed in distinct subsets of neurons. Neuron 14:497-507. CrossRef Medline

Varoqueaux F, Jamain S, Brose N (2004) Neuroligin 2 is exclusively localized to inhibitory synapses. Eur J Cell Biol 83:449-456. CrossRef Medline

Varoqueaux F, Aramuni G, Rawson RL, Mohrmann R, Missler M, Gottmann K, Zhang W, Südhof TC, Brose N (2006) Neuroligins determine synapse maturation and function. Neuron 51:741-754. CrossRef Medline

Yamagata M, Sanes JR, Weiner JA (2003) Synaptic adhesion molecules. Curr Opin Cell Biol 15:621-632. CrossRef Medline

Zhang H, Macara IG (2006) The polarity protein PAR-3 and TIAM1 cooperate in dendritic spine morphogenesis. Nat Cell Biol 8:227-237. CrossRef Medline 